\title{
Three-dimensional instabilities and transition of steady and pulsatile axisymmetric stenotic flows
}

\author{
By S. J. SHER WIN ${ }^{1}$ AND H. M. BLACKBUR ${ }^{2}$ \\ ${ }^{1}$ Department of Aeronautics, Imperial College London, South Kensington Campus, \\ London SW7 2AZ, UK \\ ${ }^{2}$ CSIRO Manufacturing and Infrastructure Technology, PO Box 56, Highett, Vic 3190, Australia
}

(Received 2 August 2004 and in revised form 13 December 2004)

A straight tube with a smooth axisymmetric constriction is an idealized representation of a stenosed artery. We examine the three-dimensional instabilities and transition to turbulence of steady flow, steady flow plus an oscillatory component, and an idealized vascular pulsatile flow in a tube with a smooth $75 \%$ stenosis using both linear stability analysis and direct numerical simulation. Steady flow undergoes a weak Coanda-type wall attachment and turbulent transition through a subcritical bifurcation, leading to hysteretic behaviour with respect to changes in Reynolds number. The pulsatile flows become unstable through a subcritical period-doubling bifurcation involving alternating tilting of the vortex rings that are ejected from the throat with each pulse. These tilted vortex rings rapidly break down through a self-induction mechanism within the confines of the tube. While the linear instability modes for pulsatile flow have maximum energy well downstream of the stenosis, we have established using direct numerical simulation that breakdown can gradually propagate upstream until it occurs within a few tube diameters of the constriction, in agreement with previous experimental observations. At the Reynolds numbers employed in the present study, transition is localized, with relaminarization occurring further downstream. A non-exhaustive investigation has also been undertaken into the receptivity of the axisymmetric shear layer in the idealized physiological pulsatile flow, with the results suggesting it has localized convective instability over part of the pulse cycle.

\section{Introduction}

The association of arterial disease with flow-related mechanisms has motivated the study of steady and pulsatile flow within both model and anatomically correct arterial model stenoses (Berger \& Jou 2000). Atherosclerosis and thrombosis are extremely important and closely linked diseases of the cardiovascular system: atherosclerosis predisposes arteries to thrombosis, and the genesis of both is intimately related to wall shear rates in the arterial flow. Atherosclerosis involves an accumulation of low-density lipoprotein (e.g. cholesterol) in the walls of the large arteries, typically where the local wall shear rate is low, and oscillatory (Caro, Fitz-Gerald \& Schroter 1971; Wootton \& $\mathrm{Ku}$ 1999). The process can be considered as a long-period nonlinear instability of the geometry of the arterial wall, wherein a local constriction can grow through promotion of flow separation. The increased pressure losses associated with flow separation can reduce the flow rate and produce ischemia (localized anoxia/absence of oxygen) which is typically used as an indicator for surgical interventions such as stenting, angioplasty and bypass operations. Over the past three to four decades there 
have been a number of published works on the topic of flow within stenotic pipes, beginning with experimental studies of flows in idealized axisymmetric stenotic tubes (Cassanova \& Giddens 1978; Khalifa \& Giddens 1981; Ahmed \& Giddens 1983, 1984; Ojha et al. 1989; Ahmed 1998). More recently, computational fluid dynamics has begun to have an impact, through numerical simulations in idealized stenoses, both axisymmetric and non-axisymmetric (Long et al. 2001; Mallinger \& Drikakis 2002), and of stenotic flows with two-dimensional planar geometries derived from MRI measurements of individual patient geometries (Stroud, Berger \& Saloner 2002). $\dagger$

Flow instability and transition to turbulence has been a common factor in these studies. Under standard physiological flow conditions, arterial flows are usually considered to be laminar, although always unsteady and often with separation. However, in the case of a stenotic pipe flow, the introduction of an inflection point into the velocity profile downstream of the contraction can lead to transition. This typically occurs in the present application for Reynolds numbers in the range of a few hundreds to a few thousands (based on upstream conditions), coinciding with those particularly relevant to physiological conditions.

The onset of turbulence has been observed in the laboratory studies, and has led to description of the flows in statistical terms. While obviously important, the descriptions have arguably not greatly aided physical understanding of the underlying fluid mechanics. This understanding could potentially be derived from numerical simulations; however, the wide variation in length and time scales involved in transitional and turbulent flows makes their numerical simulation quite demanding. This is especially true when accompanied in the present case by geometrical complexity and the need for description in terms of a number of dimensionless parameters. While the past numerical works have aided our understanding of stenotic flows, nowhere in the literature can be found a fundamental study that examines the stability of the flows, even in the simplest case of axisymmetric flow through a smooth constriction, and the nature of the subsequent transition. This is our current contribution. Having understanding of the simplest cases, we will then be in a position to provide perspective on and explain the relevance of these mechanisms in more complex configurations.

In the present work, we turn our attention to the stability of steady and pulsatile flows in an axisymmetric stenotic tube. The approach adopted is to analyse the global linear stability of the axisymmetric flows, both steady and pulsatile, to arbitrary three-dimensional perturbations. As the problem has rotational symmetry about the cylindrical axis, it is natural to use Fourier decomposition in the azimuthal direction in order to break the general three-dimensional linear stability problem into a set of twodimensional ones, dramatically reducing the size of each individual problem. Once we have the most unstable mode for each flow, we then use full three-dimensional direct numerical simulation (DNS) in order to examine the evolution of their instability modes, onset of turbulence and nonlinear dynamics.

As is commonly justified in many works on blood flows in the major arteries, Newtonian rheology has been assumed. While the effect of artery wall compliance is potentially important, it is often taken to be of secondary significance, and has been ignored in the present work. For discussion of these assumptions and reviews of recent work on blood flow in arteries, see Ku (1997), Wootton \& Ku (1999), Berger \& Jou (2000) and Taylor \& Draney (2004).

$\dagger$ For purely oscillatory flows in unconstricted straight tubes, instability mechanisms different to those reported in the current paper arise: these have been investigated by Yang \& Yih (1977) and Akhavan, Kamm \& Shapiro (1991). 


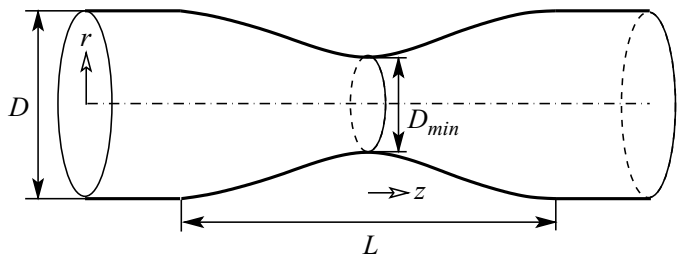

Figure 1. Geometrical parameters that define the axisymmetric sinusoidal stenosis.

The kinematic and dynamical parameters of the problem are introduced in $\S 2$. In order to keep the study to a reasonable size, our focus is restricted to a single smooth stenosis with $75 \%$ occlusion and to three simple flows: one steady, two pulsatile. In $\S 3$, we then discuss the governing equations and concepts of stability analysis adopted within this paper. In $\S 4$, we detail the numerical techniques and resolution studies undertaken to validate the results. In $\S 5$, we consider instability of steady flow, prior to introducing flow pulsatility. Section 6 covers the central topic, instability and transition in our two selected types of pulsatile flow. In that section, we also briefly examine the susceptibility of one of the pulsatile flows to axisymmetric convective shear-layer instability, using two-dimensional DNS.

\section{Problem definition: dimensionless groups}

We consider the steady and pulsatile Newtonian flow through a rigid axisymmetric stenosis described by a sinusoidal axial variation where the radius at the minimum constriction is half that of the maximum radius. For the axisymmetric geometry a $50 \%$ reduction in radius necessarily corresponds to a $75 \%$ reduction in area. This reduction has been chosen for our study based upon medical practice, where a $50 \%$ reduction in radius is typically given as the level at which a constriction can be confidently identified using medical imaging techniques such as ultrasound. Further, a $75 \%$ occlusion has also been used in many of the previous laboratory and numerical investigations.

\subsection{Geometrical parameters}

As shown in figure 1, we consider a pipe of internal diameter $D$ with an axisymmetric stenotic region with a radius $r(z)$ that varies over an axial length $L$, according to the form

$$
r(z)=0.5 D_{\text {min }}+0.5\left(D-D_{\text {min }}\right) \sin ^{2}(\pi z / L) \quad(-0.5 \leqslant(z / L) \leqslant 0.5),
$$

where $z$ is centred on the middle of the stenosis. Given this geometry, there are two independent dimensionless geometric parameters we can define which are the diameter ratio $D_{\min } / D$ and the stenosis length $\lambda=L / D$. In the clinical environment the stenosis degree, $S$, is more commonly used than the diameter ratio and is defined as the ratio of the reduction in cross-sectional area to the original area, i.e.

$$
S=1-\left(D_{\min } / D\right)^{2},
$$

In the following, we have considered a single geometry defined by $S=0.75$ and $\lambda=2$.

\subsection{Flow parameters}

To complement the geometric factors we must also consider the physiological flow parameters which describe our problem. We start by defining an axisymmetric inflow 

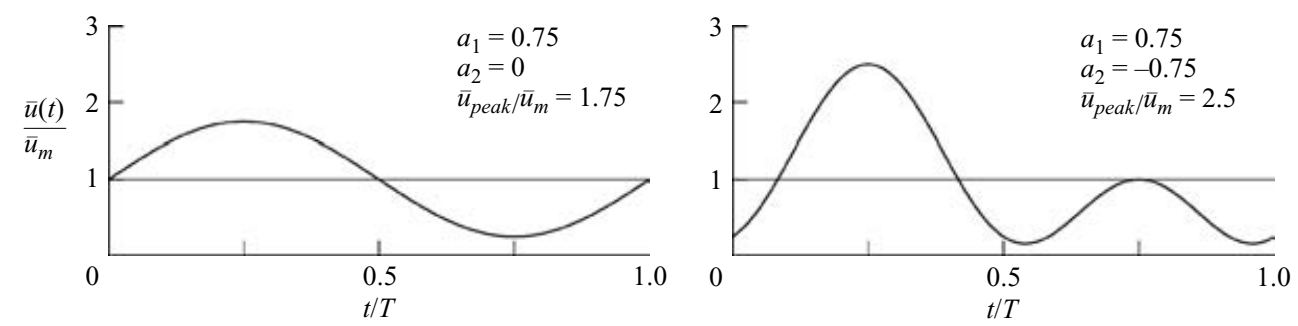

FIGURE 2. Waveforms of $\bar{u}(t)$ in the two pulsatile flow cases considered, cf. (2.2).

profile $u(r, t)$ with temporal period $T$ which has a sectional average

$$
\bar{u}(t)=\left(8 / D^{2}\right) \int_{0}^{D / 2} u(r, t) r \mathrm{~d} r
$$

and temporal average

$$
\bar{u}_{m}=\frac{1}{T} \int_{0}^{T} \bar{u}(t) \mathrm{d} t .
$$

For given $\bar{u}(t)$, we can define at least three independent dimensionless groups: the reduced velocity $U_{\text {red }}$, the peak-to-mean flow ratio, and the Reynolds number $R e$, respectively given by

$$
U_{\text {red }}=\bar{u}_{m} T / D, \quad U_{p m}=\max _{0 \leqslant t \leqslant T} \bar{u}(t) / \bar{u}_{m}=\bar{u}_{\text {peak }} / \bar{u}_{m}, \quad R e=\bar{u}_{m} D / \nu,
$$

where $v$ is the kinematic viscosity of the fluid.

Any periodic $\bar{u}(t)$ can be represented as a Fourier series. For fully developed laminar periodic flow in a circular tube, the velocity profile components at each temporal harmonic can be obtained in an analytic closed form provided by Womersley (1955). Each harmonic $n$ has an associated circular frequency $\omega_{n}=2 n \pi / T$, with the velocity distribution given through Womersley's solution in terms of a complex Bessel function as

$$
u_{n}(r, t)=\operatorname{Re}\left[\frac{A_{n} \mathrm{i}}{\rho \omega_{n}}\left(\frac{J_{0}\left(\mathrm{i}^{3 / 2} \alpha 2 r / D\right)}{J_{0}\left(\mathrm{i}^{3 / 2} \alpha\right)}-1\right) \exp \mathrm{i} \omega_{n} t\right],
$$

where $\mathrm{i}=(-1)^{1 / 2}, \rho$ is the fluid density and $\alpha=\left(\omega_{n} / v\right)^{1 / 2} D / 2$ is the Womersley number. A further input in the above expression is the value of $A_{n}$ which is a complex number representing the driving pressure gradient $\partial_{z} p=A_{n} \operatorname{expi} \omega_{n} t$ and can be determined from a specified sectional-average velocity $\bar{u}(t)$ at a given harmonic. When $n=0,(2.1)$ reduces to the standard parabolic Hagen-Poiseuille profile for steady flow.

A wide variety of pulsatile flow waveforms are observed in arteries (see e.g. Mills et al. 1970; McDonald 1974); these vary with position in the arterial system, in response to exertion, and from individual to individual. We assume that $\bar{u}(t)$ is described by the mean and two harmonic terms in the form

$$
\bar{u}(t)=\bar{u}_{m}\left[1+a_{1} \sin (2 \pi t / T)+a_{2} \cos (4 \pi t / T)\right],
$$

and for two cases of non-reversing flow: $\left(a_{1}=0.75, a_{2}=0\right)$ and $\left(a_{1}=0.75, a_{2}=-0.75\right)$, as shown in figure 2 . The first is a simple pulsatile waveform of the type considered in many laboratory experiments (Cassanova \& Giddens 1978; Ahmed \& Giddens 1984; Ojha et al. 1989), and has $U_{p m}=1.75$. The second, a more realistic approximation of physiologic waveforms since it has a higher peak-to-mean ratio, is obtained using 
two harmonics and with $a_{1}=-a_{2}=0.75$. We note that if the magnitude of the two harmonics is fixed at 0.75 , the phase relationship between them, as defined by (2.2), leads to the highest possible peak-to-mean flow ratio, $U_{p m}=2.5$.

The Womersley number, $\alpha$, can be interpreted as the ratio of the diameter (or radius) to the viscous laminar boundary-layer growth $(\nu T)^{1 / 2}$ in time period $T$. It is therefore the ratio of two sectional length scales and is a commonly used parameter in pulsatile flows of biomedical interest. We note, however, that for our problem there is also an axial length scale, associated with the stenosis shape, in the flow direction. The reduced velocity $U_{\text {red }}=\bar{u}_{m} T / D$ can be interpreted as the convective length in diameters that the mean flow moves in time $T$ and therefore introduces an axial scale into the parameter. In the following work, we will typically adopt reduced velocity, $U_{\text {red }}$, in preference to Womersley number, $\alpha$, but note that $U_{\text {red }}$ and $\alpha$ are dependent parameters related by the Reynolds number according to the relation $U_{\text {red }}=\pi R e /\left(2 \alpha^{2}\right)$.

Within this paper, we consider steady flow (i.e. $a_{1}=a_{2}=0$ ) in the Reynoldsnumber regime $400 \leqslant R e \leqslant 800$. The pulsatile flow given the most attention is the case $\left(a_{1}=0.75, a_{2}=0\right)$ for which we have considered the parametric region $2.5 \leqslant U_{\text {red }} \leqslant 7.5$ and $250 \leqslant R e \leqslant 550$. For the case $\left(a_{1}=0.75, a_{2}=-0.75\right)$ we have examined a single reduced velocity, $U_{\text {red }}=5$, over the range $500 \leqslant R e \leqslant 550$.

The Reynolds-number regime was dictated by the onset of linear instability of the steady and pulsatile flows. In terms of physiological conditions, we note that the Reynolds-number regime considered for steady flow is relatively high. For pulsatile flow, the Reynolds numbers considered are slightly high, although plausible. The Womersley numbers for the pulsatile waveforms range from $\alpha=7.2$ to 18.6, which is also physiologically reasonable; however, the peak-to-mean flow ratios are somewhat low since physiological waveforms can achieve a value of $U_{p m} \approx 4$ (McDonald 1974).

\section{Equations, stability and symmetry}

Assuming the fluid to be Newtonian and the flow incompressible, the relevant equations of motion for the primitive (velocity, pressure) variables are the incompressible Navier-Stokes equations

$$
\partial_{t} \boldsymbol{u}=-\boldsymbol{A}(\boldsymbol{u})-\nabla P+v \nabla^{2} \boldsymbol{u} \quad \text { with } \quad \nabla \cdot \boldsymbol{u}=0
$$

where $\boldsymbol{u}=\boldsymbol{u}(z, r, \theta, t)=(u, v, w)(t)$ is the velocity field, $\boldsymbol{A}(\boldsymbol{u})$ represents nonlinear advection terms, $P=p / \rho$ is the modified pressure and $v$ is the fluid's kinematic viscosity. The variables $z, r, \theta$ and $t$ are, respectively, the axial, radial, azimuthal and time coordinates and $u, v$ and $w$ are the velocity components in the axial, radial and azimuthal directions. We can consider the nonlinear terms either in convective form $\boldsymbol{A}(\boldsymbol{u})=\boldsymbol{u} \cdot \nabla \boldsymbol{u}$, conservative form $\boldsymbol{A}(\boldsymbol{u})=\nabla \cdot \boldsymbol{u} \boldsymbol{u}$, or skew-symmetric form $\boldsymbol{A}(\boldsymbol{u})=(\boldsymbol{u} \cdot \nabla \boldsymbol{u}+\nabla \cdot \boldsymbol{u} \boldsymbol{u}) / 2$, which are all equivalent in a continuum setting. Taking the pressure to represent the solution of a Poisson equation that has the divergence of the advection terms as forcing, we can consider the Navier-Stokes equations in symbolic form as

$$
\partial_{t} \boldsymbol{u}=-\left(\boldsymbol{I}-\nabla \nabla^{-2} \nabla \cdot\right) \boldsymbol{A}(\boldsymbol{u})+v \nabla^{2} \boldsymbol{u}=\boldsymbol{N}(\boldsymbol{u})+\boldsymbol{L}(\boldsymbol{u}),
$$

where the nonlinear operator $N$ contains contributions from both pressure and advection terms, while the linear operator $\boldsymbol{L}$ corresponds to viscous diffusion.

When analysing the linear stability of a flow in terms of its normal modes, we decompose the velocity $\boldsymbol{u}$ into a base flow $\boldsymbol{U}$ and perturbation flow $\boldsymbol{u}^{\prime}: \boldsymbol{u}=\boldsymbol{U}+\boldsymbol{u}^{\prime}$, and examine the stability of the perturbation linearized about the base flow. In 
this decomposition, the original nonlinear advection terms are replaced with their linearized equivalent (here for the convective form) $\partial_{\boldsymbol{U}} \boldsymbol{A}\left(\boldsymbol{u}^{\prime}\right)=\boldsymbol{U} \cdot \nabla \boldsymbol{u}^{\prime}+\boldsymbol{u}^{\prime} \cdot \nabla \boldsymbol{U}$ and in symbolic form we write

$$
\partial_{t} \boldsymbol{u}^{\prime}=\partial_{\boldsymbol{U}} \boldsymbol{N}\left(\boldsymbol{u}^{\prime}\right)+\boldsymbol{L}\left(\boldsymbol{u}^{\prime}\right)
$$

for the evolution of the linear perturbation.

If the base flow is steady in time, then so is the nonlinear operator $\partial_{U} N$. Under the assumption of normal modes, $\boldsymbol{u}^{\prime}(t) \equiv \tilde{\boldsymbol{u}} \exp \gamma t$, (3.3) becomes an eigenproblem

$$
\gamma \tilde{\boldsymbol{u}}=\left(\partial_{U} \boldsymbol{N}+\boldsymbol{L}\right) \tilde{\boldsymbol{u}},
$$

where $\gamma$ is an eigenvalue and $\tilde{\boldsymbol{u}}$ an eigenfunction, both, in general, appearing in complex-conjugate pairs. For a finite time increment $\tau$ we have

$$
\boldsymbol{u}^{\prime}\left(t_{0}+\tau\right)=\exp \left[\left(\partial_{\boldsymbol{U}} \boldsymbol{N}+\boldsymbol{L}\right) \tau\right] \boldsymbol{u}^{\prime}\left(t_{0}\right)
$$

and we usually try to extract the eigenpairs $\{\Gamma, \tilde{\boldsymbol{u}}\}$ of operator $\exp \left[\left(\partial_{U} \boldsymbol{N}+\boldsymbol{L}\right) \tau\right]$. This is because the numerical method to be used, extracts dominant eigenvalues, i.e. those of largest modulus, whereas for the steady flow we are ultimately interested in values of $\gamma$ with the largest real part, i.e. the most unstable. There is a direct correspondence between the dominant values of $\Gamma$ and the most unstable values of $\gamma$ through the relation $\Gamma=\exp \gamma \Delta t$

If, on the other hand, the base flow is time-periodic with period $T, \partial_{U} N$ is still linear but now time-periodic, and

$$
\boldsymbol{u}^{\prime}\left(t_{0}+T\right)=\exp \left[\int_{t_{0}}^{t_{0}+T}\left(\partial_{U} \boldsymbol{N}+\boldsymbol{L}\right) \mathrm{d} t\right] \boldsymbol{u}^{\prime}\left(t_{0}\right)
$$

The eigenpairs of this Floquet problem are $\left\{\mu, \tilde{\boldsymbol{u}}\left(t_{0}\right)\right\}$ where $\mu$ is a (constant) Floquet multiplier and $\tilde{\boldsymbol{u}}\left(t_{0}\right)$ is the $T$-periodic Floquet eigenfunction, evaluated at phase $t_{0}$. The equivalent to the eigenvalues $\gamma$ of the time-invariant case are the Floquet exponents $\sigma$, related to the multipliers by $\mu=\exp \sigma T$. Floquet modes are unstable for $|\mu|>1$, i.e. when Floquet multipliers leave the unit circle in the complex plane. Again, in general, the Floquet multipliers/exponents and eigenfunctions occur in complexconjugate pairs. Three primary instability scenarios can be identified: $(i)$ the critical Floquet multiplier $\mu_{c}=+1$, giving a synchronous instability with period $T$ at onset; (ii) $\mu_{c}=-1$, giving a period-doubling bifurcation; (iii) $\mu_{c}=\exp \pm \mathrm{i} \phi$, a NeimarkSacker bifurcation, which introduces a new secondary period and gives quasi-periodic solutions.

It is readily apparent that the structure of (3.5) and (3.6) is the same, except that in the former case the time-invariant operators have been taken outside the integral. The conceptual difficulties of numerically constructing the exponential operators are reduced once it is appreciated that the action of these operators can be evaluated by standard time-integration techniques applied to the linearized evolution equation (3.3).

Since the geometry is axisymmetric, the velocity must be $2 \pi$-periodic in $\theta$ and can be projected exactly onto a set of two-dimensional complex Fourier modes by

$$
\hat{\boldsymbol{u}}_{k}(z, r, t)=\frac{1}{2 \pi} \int_{0}^{2 \pi} \boldsymbol{u}(z, r, \theta, t) \exp (-\mathrm{i} k \theta) \mathrm{d} \theta
$$


where $k$ is an integer wavenumber. This projection has the Fourier series reconstruction

$$
\boldsymbol{u}(z, r, \theta, t)=\sum_{k=-\infty}^{\infty} \hat{\boldsymbol{u}}_{k}(z, r, t) \exp (\mathrm{i} k \theta)
$$

The Fourier-transformed equations of motion and axial boundary conditions for the velocity and pressure (and their perturbations) in cylindrical coordinates are described in detail by Blackburn (2002) and Blackburn \& Sherwin (2004). Our base flows are both axisymmetric/two-dimensional, i.e. $\widehat{\boldsymbol{U}}_{k}=\mathbf{0}, k \neq 0$, and two-component, i.e. $\boldsymbol{U} \equiv(U, V, 0)$. In the numerical stability analysis, we take advantage of linearity, which decouples the stability problem for each $\hat{\boldsymbol{u}}_{k}^{\prime}$.

The axisymmetric base flows have rotational symmetry about the $z$-coordinate axis, i.e. the spatial symmetry group of the base flows is $O(2)$, broken by any threedimensional instability. This has consequences for the possible forms of threedimensional instabilities. If the eigenvalues/Floquet multipliers of the instability modes are real, then the bifurcations occur through a pitchfork of revolution, i.e. the mode shapes are aligned at arbitrary, but fixed, azimuthal coordinate in the tube; an azimuthal traverse corresponds to a revolution of the pitchfork. For complex-conjugate pair eigenvalues there are two cases to consider: standing-wave type solutions, which again bifurcate through a pitchfork of revolution, producing mode shapes at arbitrary, but fixed, azimuthal coordinates, and travelling-wave (or, in this system, rotating-wave) type solutions that bifurcate in $\theta$-conjugate pairs, one rotating in $\mathrm{a}+\theta$ sense, the other in a $-\theta$ sense: only one of the pairs will be observed in practice. In this case, the imaginary part of the eigenvalue/multiplier is associated with the precession speed of the rotating wave. At linear onset, the eigenvalues for the standing- and rotating-wave solutions are identical, and the mode shapes of the standing waves are symmetric combinations of those for the rotating waves. Under nonlinear evolution (i.e. DNS), however, the standing and rotating wave solution branches are distinct, and at most one has stable solutions.

In order to account for the various possibilities, we initially use perturbation velocities with restricted symmetry, and also arbitrarily align the mode shape at $\theta=0$ :

$$
\left(u^{\prime}, v^{\prime}, w^{\prime}\right)(z, r, \theta, t)=\left(u^{\prime} \cos k \theta, v^{\prime} \cos k \theta, w^{\prime} \sin k \theta\right)(z, r, t),
$$

which corresponds to taking $\hat{u}_{k}^{\prime}$, $\hat{v}_{k}^{\prime}$ purely real and $\hat{w}_{k}^{\prime}$ purely imaginary. This combination passes unaltered through the linearized Navier-Stokes equation when the base flow is two-component, and is sufficient except when the eigenvalues are complexconjugate pairs, in which case full-complex perturbations must also be introduced, in order to capture the shape of the travelling-wave modes (see e.g. Blackburn \& Lopez $2003 a, b$ ). In fact, for the present problem, we have so far only observed instability modes with real eigenvalues, so the above form is adequate. When carrying out DNS studies, the full complex structure of all modes is used, removing the symmetry constraint implicit in (3.9).

\section{Numerical techniques}

The same underlying spatial and temporal discretizations were applied both to integrate the Navier-Stokes equations (3.1) in direct numerical simulation (DNS), and their linearized equivalents used to evolve perturbation velocities (3.3). These numerical methods are outlined in $\S 4.1$ and this is followed in $\S 4.3$ by those relevant to stability analysis techniques. 
(a)

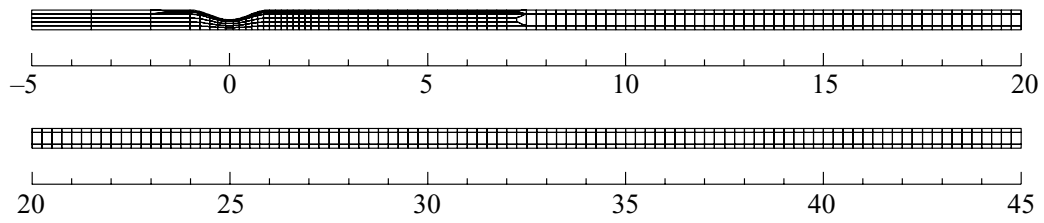

(b)

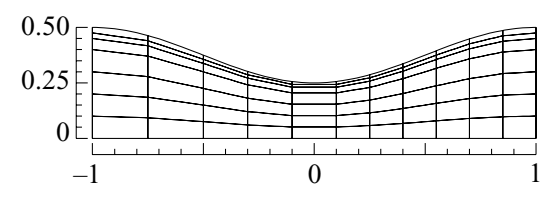

(c)
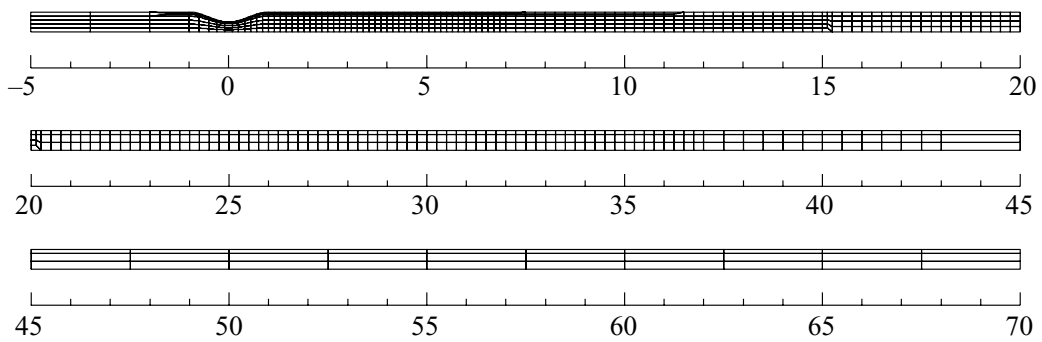

FIGURE 3. Spectral element outlines of computational mesh, dimensions given in terms of tube diameter $D$. (a) Elements for the first mesh with an outflow at $45 D$. (b) A close-up of the throat, with curved element edges. $(c)$ The extended mesh with an outflow at $70 D$ and is similar to the mesh in $(a)$ up to $z / D=7$.

\subsection{Treatment of the evolution equations}

For time evolution of both the full and linearized Navier-Stokes equations, we rely on standard (nodal-Gauss-Lobatto-Legendre) spectral elements in $(z, r)$ and Fourier expansions if required in the azimuthal $\theta$-direction. This spatial discretization was coupled with a second-order-time velocity correction time-integration scheme. The development of this numerical method for DNS has been described in detail by Blackburn \& Sherwin (2004), where full spectral convergence in both the meridional semi-plane and in azimuth was demonstrated. The application of the method to linearized Navier-Stokes evolution, including appropriate boundary conditions, has also previously been described by Blackburn (2002). As a consequence, we refer the interested reader to these other publications for more detail on the numerical simulation techniques employed.

One aspect we highlight here concerns the discrete formulation of the advection terms. We have found that for three-dimensional cylindrical-coordinate DNS, the skew-symmetric form $\boldsymbol{A}(\boldsymbol{u})=(\boldsymbol{u} \cdot \nabla \boldsymbol{u}+\nabla \cdot \boldsymbol{u} \boldsymbol{u}) / 2$ provides superior numerical stability to the convective form $\boldsymbol{A}(\boldsymbol{u})=\boldsymbol{u} \cdot \nabla \boldsymbol{u}$, and have used it exclusively in that application. On the other hand, we use the convective form and its linearized equivalent for computing two-dimensional/axisymmetric flows and evolution of linearized perturbations for stability analysis. The use of convective form in the stability analysis is consistent with the work of Wilhelm \& Kleiser (2001) who found that this form does not lead to numerical linear instability in spectral element type discretizations.

\subsection{Mesh layout and resolution}

The computational meshes used in the following numerical calculations are shown in figure 3. The first domain used for the majority of calculations is shown in 
figure 3(a) and consists of 743 elemental regions. In each element two-dimensional mapped tensor-product Lagrange-interpolant shape functions based on the GaussLobatto-Legendre nodes were applied. At polynomial order $N_{p}=7$, this elemental discretization corresponds to approximately 38000 local degrees of freedom in each meridional semiplane. The domain extended $5 D$ upstream and $45 D$ downstream of the throat. As shown in figure $3(b)$, a fine radial mesh spacing was adopted in the region of the stenosis where two layers each of $5 \%$ of the local radius were applied. At $z / D \approx 7$, the radial mesh spacing was coarsened to allow a uniform axial spacing of $0.5 D$ to be applied to the outflow. For the two harmonic waveforms where $a_{1}=0.75, a_{2}=-0.75$ in (2.2), we required a domain with a longer outflow as shown in figure $3(c)$. This mesh contains 915 elemental regions and when $N_{p}=9$ this corresponds to approximately 91000 local degrees of freedom in each meridional semiplane. The two meshes are similar up to $z / D \approx 7$, the second mesh then has radial transitions at $z / D \approx 11,15$ and 20 .

The long outflow lengths of both meshes were found to be necessary primarily to obtain satisfactory convergence of the pulsatile flow stability analyses. As will be seen later, the linear instability modes for both steady and pulsatile flows are significantly extensive in the axial direction. The need for this apparently extreme domain length can be understood heuristically by considering a case where $U_{\text {red }}=7.5$ with a stenosis of $S=0.75$. For this problem there is a fourfold increase in the sectionally averaged velocity at the throat of the stenosis and so in the absence of any resistive force this flow could theoretically propagate through a length of order $4 \times 7.5 \mathrm{D}=30 \mathrm{D}$ in one pulsatile cycle. A further significant feature of the mesh design was the need to adequately resolve shear layers, both next to the wall of the stenosis, and as they propagate in the elongated separation zone further downstream. The fine resolution around the stenosis where the highest sectional flow rates are observed, results in a reasonably restrictive time step dictated by CFL stability considerations. This restriction arises owing to the explicit treatment of advection terms adopted in the numerical integration of the Navier-Stokes equations (Blackburn \& Sherwin 2004).

Ultimately the spectral element layout and polynomial order adopted in the meridional semiplane was decided on the basis of satisfactory convergence of stability analysis results. We have found in past work (e.g. Blackburn 2002; Blackburn \& Lopez $2003 b$ ) that mesh designs which deliver adequate convergence in stability analyses also do so for DNS studies, consistent with points made by Zang (1991). Typical resolution results will be provided in the following section. However, prior to carrying out these studies, and all other stability analyses, it was always necessary to ascertain that the base flows in question were either sufficiently close to an asymptotic state (for steady flows) or a periodic one (for pulsatile flows). In all the base-flow calculations, flow time series data were extracted at $z / D=0,1,22,43$ and $r / D=0.25$. For steady flows, the base flow was considered to be close enough to an asymptotic state when the velocities converged to the local steady-state value within a relative error of order $1 \times 10^{-4}$. In practice, this typically required a total run time of order $t \bar{u}_{m} / D=75$ after any parameter change. For the pulsatile computations, the base flow was run for a sufficient number of cycles so that the mean flow had time to convect through the entire computational domain. For example at $U_{\text {red }}=5.0$, the flow was run for 10 cycles after any parameter change. Phase-plane plots of the $z$-component versus $r$-component velocities at each extraction point were then checked for the final two cycles to ensure time periodicity of the flow within visual limits, again to a typical relative accuracy of order $1 \times 10^{-4}$ or better. It should be noted that through these methods of computing and checking the base flows, we have established that none 


$\begin{array}{cc}N_{p} & N_{\text {tot }} \\ 4 & 12673 \\ 5 & 19556 \\ 6 & 27925 \\ 7 & 37780 \\ 8 & 49121\end{array}$

\begin{tabular}{cccc}
\multicolumn{2}{r}{$\begin{array}{r}R e \\
|\gamma|\end{array}$} & & \multicolumn{2}{c}{$\operatorname{Re}=400, U_{\text {red }}=2.5$} \\
& $\theta$ & $|\mu|$ & $\theta$ \\
$382.73 \times 10^{-3}$ & \pm 2.0554 & 1.4463 & \pm 2.5749 \\
$9.1686 \times 10^{-3}$ & 0 & 1.1444 & $\pi$ \\
$8.9666 \times 10^{-3}$ & 0 & 1.0726 & $\pi$ \\
$8.9624 \times 10^{-3}$ & 0 & 1.0731 & $\pi$ \\
$8.9571 \times 10^{-3}$ & 0 & 1.0736 & $\pi$
\end{tabular}

TABLE 1. Convergence of eigenvalue $\gamma$ or Floquet multiplier $\mu$ of the leading $k=1$ azimuthal Fourier mode as a function of polynomial order $N_{p}$, for both steady and pulsatile flow. At each $N_{p}, N_{\text {tot }}$ is the number of independent mesh points for the spectral element mesh of figure $3(a)$.

possesses an axisymmetric absolute instability mode for clean inflow conditions. In $\S 6.4 .3$, it will be demonstrated that a convective shear-layer instability is possible when high-frequency forcing is introduced into the inflow.

\subsection{Stability analysis methodology}

The numerical methods employed for stability analysis of both steady and pulsatile flow follow those outlined in Tuckerman \& Barkley (2000), and previously described and used in other works (e.g. Barkley \& Henderson 1996). The code used in the present study has been validated against the circular cylinder wake secondary stability analysis of Barkley \& Henderson, see Blackburn \& Lopez (2003a), and against experimental and DNS results for oscillatory swirling flows (Blackburn 2002). For both steady and time-periodic flows, the analysis is based on a Krylov-subspace iteration of successive finite increments of (initially random) perturbations through the linearized equations (3.5) and (3.6) using an Arnoldi method to extract the dominant eigenpairs of the exponential operators in the equations. For Floquet analysis, the data used to supply the $T$-periodic base flow are approximated through Fourier-series reconstruction from a limited number (typically 256) of time-slices obtained from two-dimensional DNS. This number of time-slices is somewhat higher than employed in previous applications (typically 32 or 64 ), as the dimensionless frequency content of the base flows here is somewhat broader.

\subsection{Resolution studies}

An identical spectral element mesh to that adopted for computing base flows (see figure $3 a$ ) was used for the steady and Floquet stability analysis with a single harmonic waveform. The tolerance placed on the Arnoldi iteration residual (see e.g. Saad 1992) as a stopping criterion was $1 \times 10^{-6}$. To determine the appropriate polynomial order for the base flow and also our stability analyses a series of convergence tests were performed. In table 1, we show the results of a polynomial-order convergence test for the three-dimensional instability in the $k=1$ azimuthal Fourier modes. Two cases are considered. The first involved the sensitivity of the leading eigenvalue in the steady-flow stability analysis at $R e=800$. The second demonstrates the sensitivity of the Floquet multiplier for analysis at $U_{\text {red }}=2.5$ and $R e=400$. Both these test cases are quite close to the onset of instability for the relevant flows. From this table, we observe that for a polynomial order of $N_{p}=7$, the variation of the eigenvalue obtained by incrementing the polynomial degree by one is of order $1 \times 10^{-4}$ and the variation of the multiplier is of order $1 \times 10^{-3}$. In the two-harmonic waveform 

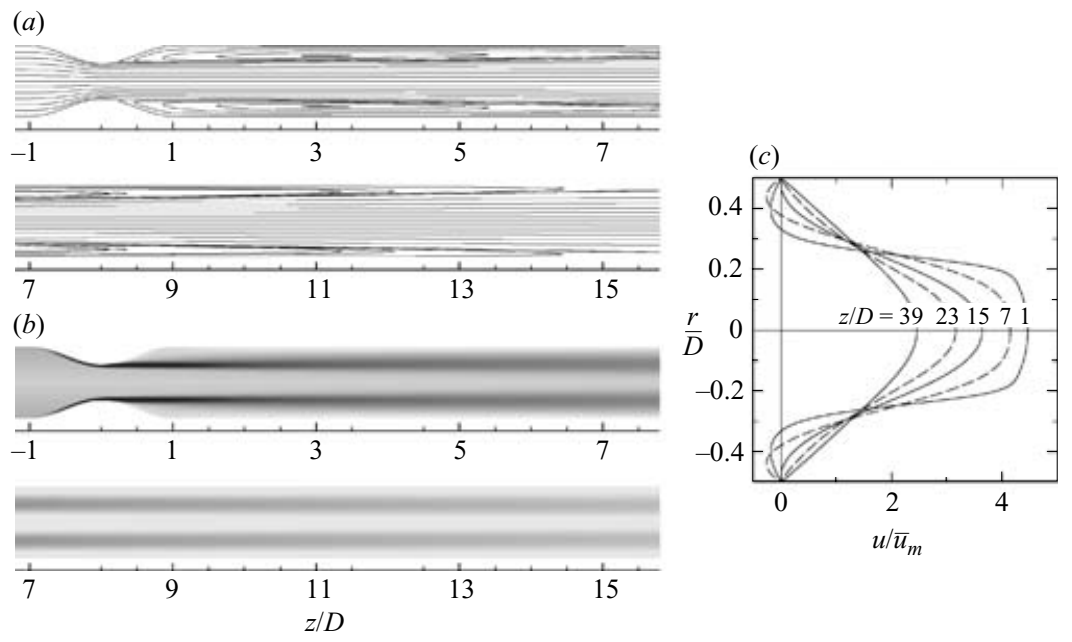

FIGURE 4. (a) Streamlines, $(b)$ vorticity contours and (c) velocity profiles for the steady base flow at $R e=750$. Reattachment occurs at $z / D=16.2$.

case (not shown), the variation of the multiplier near criticality was also of order $1 \times 10^{-3}$ when changing from $N_{p}=9$ to $N_{p}=10$.

Additional resolution checks were also performed at $U_{\text {red }}=7.5, R e=500$ where similar spatial convergence was observed. As a result, $N_{p}=7$ was chosen for all subsequent investigations, except for the two-harmonic case, where we have used $N_{p}=9$. Finally, time-step independence checks were also performed at $U_{\text {red }}=2.5, R e=400$, $N_{p}=7$ and $k=1$, by considering values of $\Delta t \bar{u} / D=1 / 7168,1 / 9216$ and $1 / 12288$; changing the time step had no effect on the computed value of $\mu$ to four-figure accuracy.

\section{Steady inlet flow}

\subsection{Base flow characteristics}

The main characteristics of the steady base flow are indicated in figure 4 where we show streamlines, vorticity contours and representative velocity profiles for the flow at $R e=750$. From mass flow considerations in the $75 \%$ stenosis, it is evident that the sectionally averaged velocity at the throat of the stenosis reaches a values of $4 \bar{u}_{m}$. The flow is unable to remain attached during the stenosis expansion and a constrained laminar jet is formed in the centre of the pipe, as highlighted by the vorticity contours in figure $4(b)$. The axisymmetric jet establishes a recirculation region immediately past the stenosis, as indicated by the streamlines, which extends downstream to approximately $z / D=16.2$. Under the action of viscous diffusion, the central core of the jet then decays back to the Hagen-Poiseuille solution. The velocity profiles of figure $4(c)$ show the axial velocity distribution as a function of the radius at fixed axial locations of $z / D=1,7,15,23$ and 39. From this plot we observe that even at $z / D=39$ the Poiseuille solution is not fully re-established, as the centreline velocity is $20 \%$ higher than its asymptotic value of $2 \bar{u}_{m}$.

\subsection{Stability analysis}

Figure 5 shows the (real) leading eigenvalue, $\gamma$, as a function of azimuthal wavenumber for Reynolds numbers 500, 600, 700 and 800. The first azimuthal Fourier mode $(k=1)$ is the least stable. An inset shows the variation of $\gamma$ with $\operatorname{Re}$ for $k=1$; the critical 


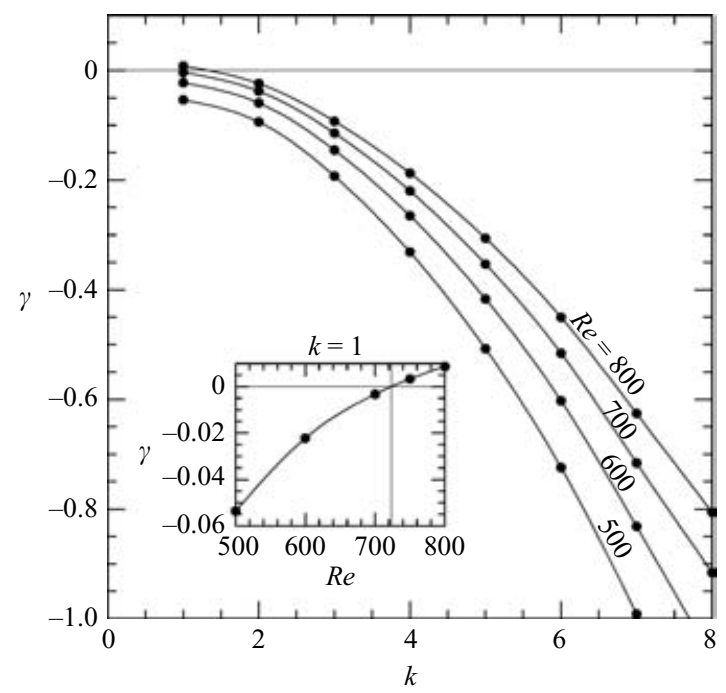

FIGURE 5. Stability analysis of steady flow: the variation of the leading eigenvalue $\gamma$ with azimuthal wavenumber $k$ for different Reynolds numbers. The critical Reynolds number, $R e_{c}=722$, is established by interpolation (inset).

Reynolds number for growth of the linearized perturbation is found to be $R e_{c}=722$, which is based on a cubic spline fit through the data.

\subsection{Direct numerical simulation}

In order to study the nonlinear behaviour of the instability, a small perturbation flow component derived from the leading instability mode is added to an unstable base flow, and the combination is evolved to saturation. Results for $R e=750$, approximately $4 \%$ greater than $R e_{c}$, are shown in figure 6 . The diagnostic used in this plot is the dimensionless amount of flow kinetic energy contained in each azimuthal Fourier mode $k$ :

$$
E_{k}=\frac{1}{2 A \bar{u}_{m}^{2}} \int_{A} \hat{\boldsymbol{u}}_{k} \cdot \hat{\boldsymbol{u}}_{k}^{*} r \mathrm{~d} A,
$$

where $A$ is the area of the two-dimensional meridional semiplane and $\hat{\boldsymbol{u}}_{k}^{*}$ denotes the complex conjugate of the velocity data in the $k$ th Fourier mode. The number of azimuthal Fourier modes chosen for this calculation was $N_{k}=32$ (64 planes of data in azimuth), which is considered to be adequate on the basis that after saturation, the energies in the non-axisymmetric modes span approximately three orders of magnitude. The total number of mesh points for this simulation is approximately 2.4 million.

The dotted line in figure 6 indicates the exponential growth rate for $k=1$, predicted from the linear stability analysis and is in close agreement with the DNS results. Note that while the initial growth in the three-dimensional perturbation is exponential, for $t \bar{u}_{m} / D \gtrsim 400$ it becomes faster than exponential prior to saturation, indicating that the bifurcation is of subcritical type. We will return to this point in $\S \S 5.4 .2$ and 5.4.3.

\subsection{Discussion}

\subsubsection{Characteristics of the linear instability mode}

In figure 7, we see features of the most unstable eigenmode (at $k=1$ ) for $R e=750$. The eigenmode arises downstream of the throat of the stenosis, and is elongated in 


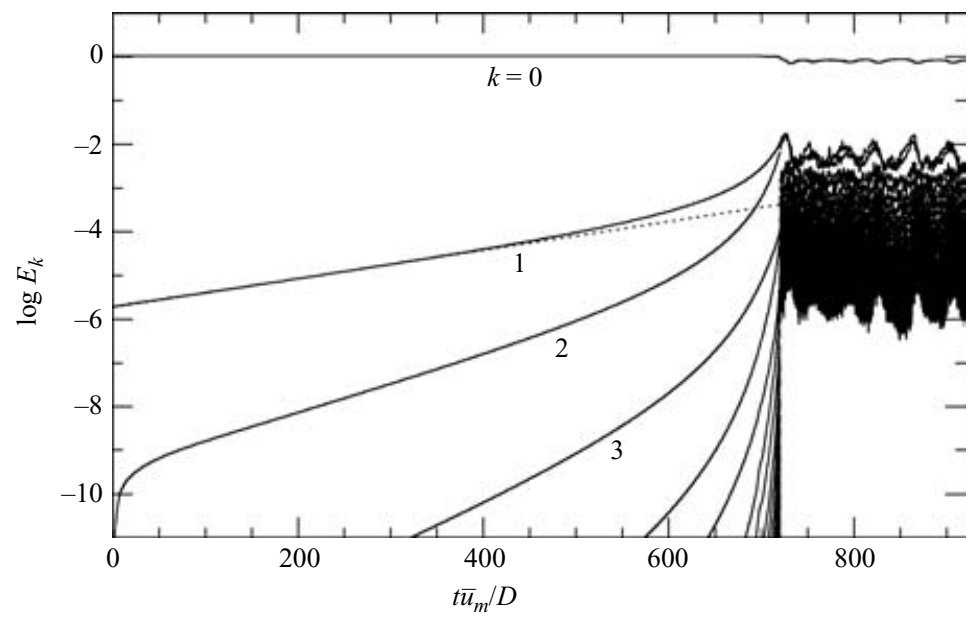

FiguRE 6. Growth to saturation and transition to turbulence of the steady inlet flow solution at $R e=750$, approximately $4 \%$ above $R e_{c}$, represented by kinetic energies in azimuthal Fourier modes, with $N_{k}=32$. The dotted line through the initial data for $k=1$ indicates exponential growth corresponding to the leading eigenvalue.

(a)
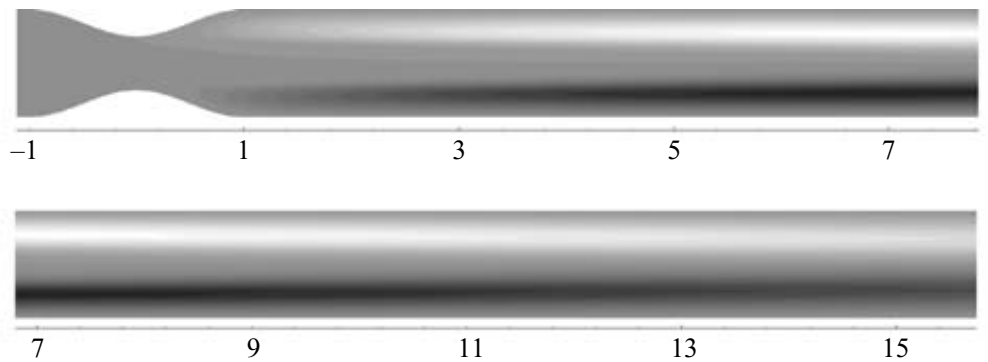

$z / D$

(b)

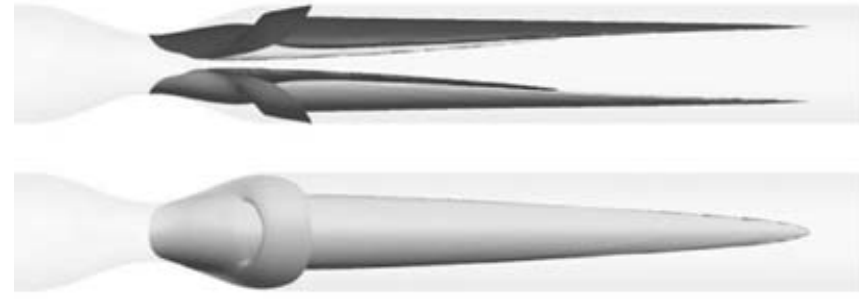

(c)

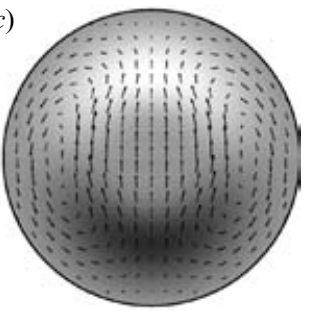

(d)

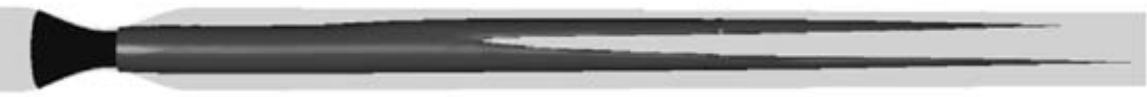

Figure 7. Most unstable eigenmode of the steady flow at $R e=750$. (a) Contours of axial velocity (white positive, black negative), $(b)$ top and side views of positive and negative isosurfaces of streamwise vorticity, $(c)$ velocity vectors of the in-plane flow superimposed on contours of streamwise velocity for a slice at $z / D=5,(d)$ isosurface of azimuthal vorticity for a linear combination the base flow and the most unstable eigenmode. 
the axial direction. The perturbation axial velocity (figure $7 a$ ) is positive at the top of the tube and negative at the bottom, which is accompanied by much weaker crossflow perturbation velocities, shown at $z / D=5$ (figure $7 c$ ). Isosurfaces of streamwise perturbation vorticity (figure $7 b$ ) reveal a quadrupole arrangement that reaches upstream to the throat of the stenosis, where the base flow separates from the wall. Finally, figure $7(d)$ shows an isosurface of azimuthal vorticity of a linear combination of the base flow and the most unstable eigenmode.

While the strongest perturbation pressures (not shown) produce a downward force just past the separation line within the stenosis which mainly affects flow in the recirculation region, further downstream, the axial perturbation velocities induce a weak cross-flow pressure gradient and flow, as demonstrated in figure $7(c)$. Therefore the perturbation flow tends to deflect the centreline of the enclosed jet away from the tube's axis of symmetry, promoting a mild Coanda-type attachment. Although the first symmetry-breaking transition in a two-dimensional expansion or constriction is also of a Coanda-type instability, see Sobey \& Drazin (1986), in the two-dimensional channel, the instability occurs at far lower Reynolds number $(O(10))$ and is far stronger in its deflection.

We also recall that the azimuthal orientation of the mode is driven by the use of a particular expansion basis (3.9), and that the mode shown is one instance of the azimuthally symmetric pitchfork of bifurcating modes.

\subsubsection{Nonlinear saturation}

As observed in $\S 5.3$, the growth to saturation in the three-dimensional modes (see figure 6) is initially faster than exponential, which becomes increasingly apparent for $t \bar{u}_{m} / D \gtrsim 400$. The implication is that the normal form for the bifurcation is (to fifth order) of type

$$
\frac{D}{\bar{u}_{m}} \frac{\mathrm{d} \psi}{\mathrm{d} t}=\gamma \psi+\beta_{1}|\psi|^{2} \psi-\beta_{2}|\psi|^{4} \psi
$$

where $\psi$ represents the amplitude of the instability mode, $\gamma$ is the linear growth rate, $\beta_{i}$ are positive constants, and that the bifurcation is subcritical. We can therefore expect hysteretic behaviour with respect to changes in Reynolds number near the critical value.

The non-axisymmetric modal energies in figure 6 are related to the squared amplitude of the perturbation $\psi$. In figure 8 , the growth in $E_{1}^{1 / 2}$ is plotted as representing $\psi$, together with the exponential growth corresponding to the leading eigenvalue derived from the linear stability analysis, and that of a nonlinear model of form (5.2), with fitted values of $\beta_{1}$ and $\beta_{2}$. It is seen that up until the onset of transition, the normal form for the bifurcation represents the perturbation growth well. Using (5.2), the computed Re-dependence of $\gamma$ (see inset, figure 5), and the fitted values of $\beta_{1}$ and $\beta_{2}$ (taken to be $R e$-independent), an approximate bifurcation diagram can be computed - this is shown as an inset to figure 8 .

\subsubsection{Turbulent flow}

Following saturation, the flow becomes highly unsteady and undergoes local transition to turbulence. This is indicated by the variation in the axisymmetric component of kinetic energy (which on a linear scale has a variation of approximately $\pm 5 \%$ about the mean value), and also in the the non-axisymmetric components, which have proportionately much larger fluctuations: all non-axisymmetric modes exhibit large fluctuations on a time scale $\Delta t \bar{u}_{m} / D \sim 35$. 


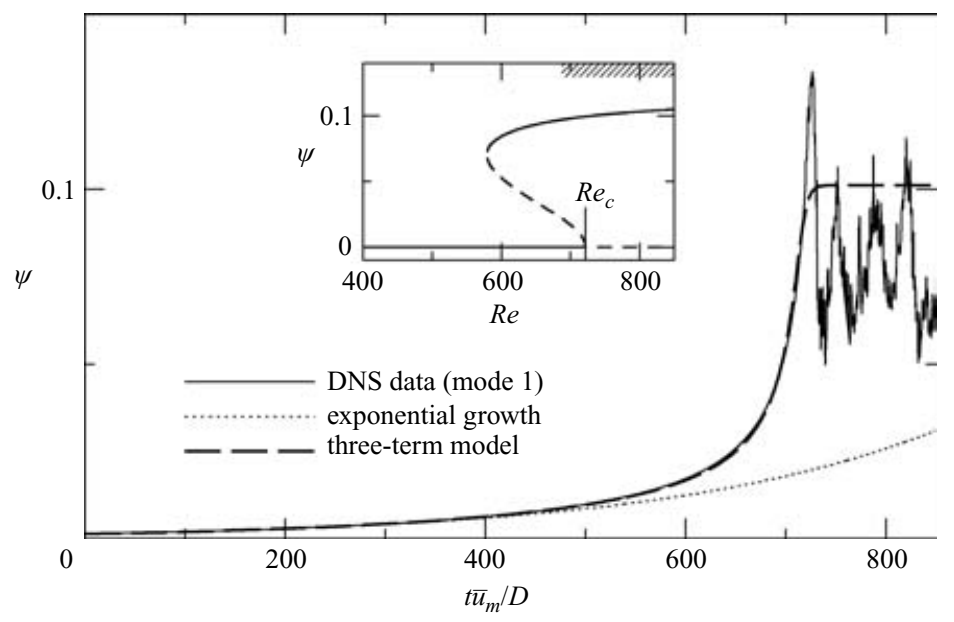

FiguRE 8. Growth to saturation and transition to turbulence of the steady inlet flow solution at $R e=750$, where $E_{1}^{1 / 2}$ (taken from figure 6) is used as a measure of perturbation amplitude $\psi$. The three-term model fit is for (5.2). Inset: approximate bifurcation diagram, with observed $R e$-range of turbulent solutions indicated by hatching.

In order to illustrate the nature of the final state of the flow and the mechanics of these long time-scale fluctuations, we show in figure 9 a set of visualizations of instantaneous vorticity isosurfaces, computed at equal time intervals over $\Delta t \bar{u}_{m} / D=35$, together with a plot indicating the time series of energy in azimuthal modes $k=0$ and $k=1$. The elongated isosurfaces that reach upstream into the stenosis are of azimuthal vorticity: these serve to indicate the location and structure of the jet's shear layer. At the initial time (figure $9 a$ ), the jet is deflected towards the top of the tube. At approximately $5 D$ downstream of the stenosis (i.e. at $z / D \approx 5$ ), there are signs of shear-layer oscillation; at $z / D \approx 10$ there is a turbulent breakdown. From the time series, it can be seen that the energy in the $k=1$ mode is about to reach a maximum at this instant. In figure $9(b)$, the turbulent breakdown has progressed upstream to lie at $z / D \approx 4$, and the time series shows that the energy in the $k=1$ mode is falling. In figures $9(c)-9(f)$, the turbulent patch washes downstream. At figure $9(g)$, the energy in the $k=1$ mode is again near a maximum, the turbulent burst begins at $z / D \approx 10$, and shear-layer oscillations are evident. However, the jet's deflection is now towards the bottom of the tube. Thus the long time-scale oscillations are associated with a flapping of the jet, accompanied by cyclic axial movements in the location of its turbulent breakdown.

As we have mentioned above, the nature of growth to saturation in the nonaxisymmetric modes at $R e=750$ indicates that the bifurcation to three-dimensional behaviour is subcritical, and we can then expect there to be hysteretic behaviour with respect to changes in Reynolds number near the critical value 722. Additional DNS runs have been made, commencing with the asymptotic/turbulent state at $R e=750$, in which the Reynolds number is decremented in steps, followed at each level by passage of sufficient simulation time for the flow state to be assessed. Following this method, we have found that the flow remains three-dimensional and turbulent down to $R e \approx 688$, less than the linear critical value, confirming the presence of hysteresis - the lower limit is indicated by the hatched region at the top of the inset in figure 8. Prior to the return to axisymmetry, the turbulent behaviour becomes more 


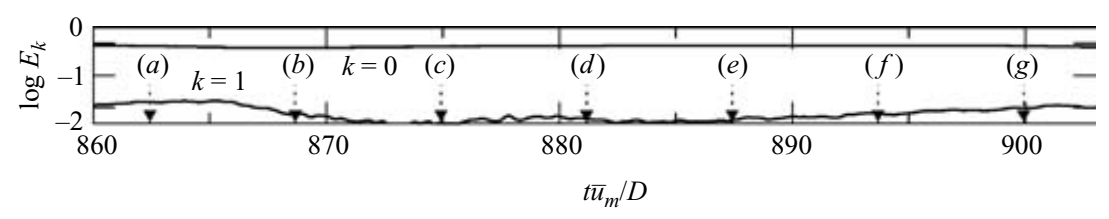

(a)

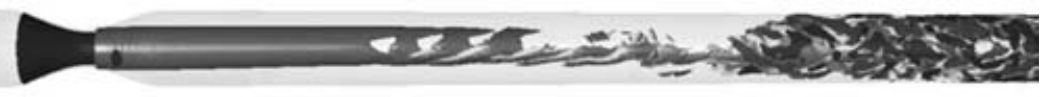

(b)

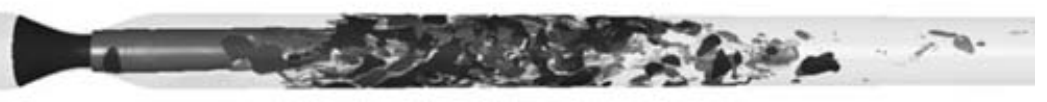

(c)

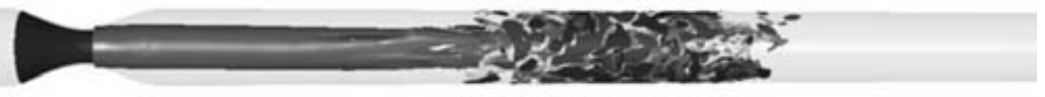

(d)

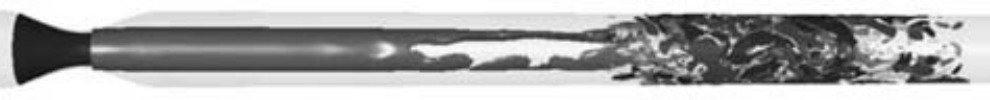

(e)

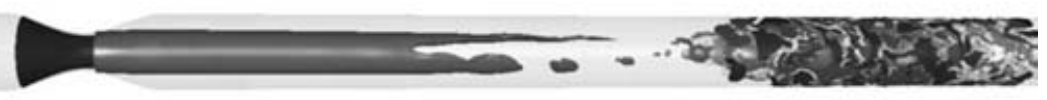

(f)

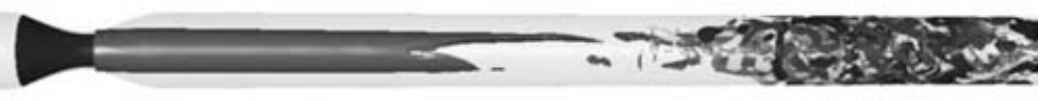

(g)

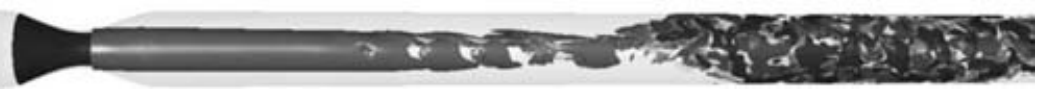

FIGURE 9. Time series of energies in the first two azimuthal Fourier modes for steady inlet flow at $R e=750$ during a long-period oscillation, and instantaneous isosurfaces of azimuthal and streamwise vorticity at seven points in the cycle. At the start of the cycle, the orientation of the jet is towards the top of the tube; at the end, it is towards the bottom.

burst-like, with greater (as much as a factor of ten) variation in the energy in the non-axisymmetric modes during any one of the long time-scale fluctuations, with each peak followed by a rapid fall in the $k=1$ and $k=2$ modes. Eventually with reducing Reynolds numbers, these decreases become terminal and the flow re-laminarizes.

\subsubsection{Comparison to previous experimental studies}

Our observations are consistent with the experimental results obtained for steady flow by Cassanova \& Giddens (1978) and Ahmed \& Giddens (1983). Cassanova \& Giddens considered both 'contoured' and 'sharp edged' stenoses. Their inflow condition was provided by a smoothly converging inlet which created a 'velocity profile entering the occlusion [which] was flat over $80 \%$ of the tube diameter' (i.e. inconsistent with fully developed Poiseuille flow), and the contours of the stenoses were composed of intersecting circular arcs. They report flow disturbances downstream of a $75 \%$-occlusion smooth stenosis at $R e=635$, although in their figure 3 the 'disturbances' shown in the first few diameters downstream of the stenosis appear to be more consistent with shear-layer oscillations than with developed turbulence.

The experiments with steady flow in Ahmed \& Giddens (1983) employed smooth stenoses with fully developed Poiseuille inflow. When considering a $75 \%$-occlusion cosine stenosis with a stenosis length of $\lambda=2$ (i.e. the same as considered here), narrowband discrete frequency oscillations were noted at $R e=500$. At $R e=1000$ 
(a)
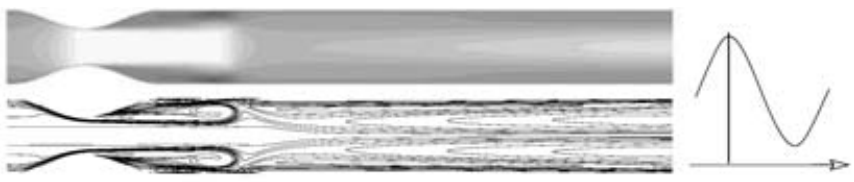

(b)
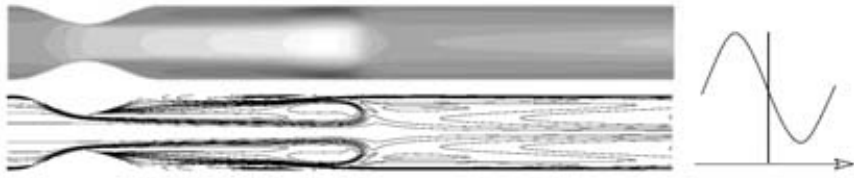

(c)
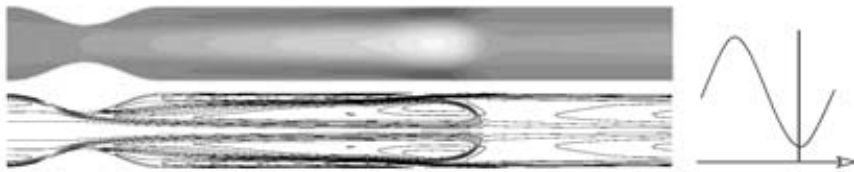

(d)
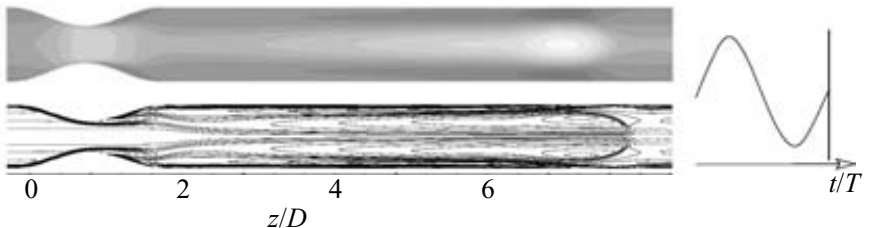

FIGURE 10. Streamwise velocity and azimuthal vorticity in the meridional plane of the axisymmetric base flow at four stages of the single harmonic pulsatile cycle when $U_{\text {red }}=2.5$, $R e=400(\alpha=15.9)$.

however, discrete oscillations were observed in the region $z / D<4$ while for $z / D>4$ turbulence occurred. As for Cassanova \& Giddens (1978), critical Reynolds numbers are not recorded.

A reasonable interpretation of these results, taken as a whole, is that for stenotic geometries similar to or the same as those that we have employed, the onset of turbulence occurred between $R e=500$ and $R e=1000$, and could propagate upstream to $z / D \approx 4$, consistent with our results. Examination of the visualization data suggests that the 'discrete frequencies' are very likely associated with shear-layer oscillations, which may have been triggered by upstream noise, and which of course is absent in our simulations.

\section{Pulsatile flow}

Having dealt with instability of the steady flow, we now turn to consider singleand two-harmonic pulsatile flows (see figure 2).

\subsection{Base flow characteristics}

\subsubsection{Single harmonic waveform}

For a single harmonic pulsatile waveform, within the Reynolds number and reduced velocity range considered, the base flow characteristics were similar to those shown in figure 10. In this figure we plot the streamwise velocity and azimuthal vorticity contours over the same range at four instants in the sinusoidal cycle. The figure includes the axial range $-1 \leqslant z / D \leqslant 7.5$ and is at a Reynolds number of $R e=400$ and reduced velocity of $U_{\text {red }}=2.5(\alpha=15.9)$. As indicated by the time trace on the right-hand side of figure 10, the instants considered correspond to the maximum, mean 
(a)

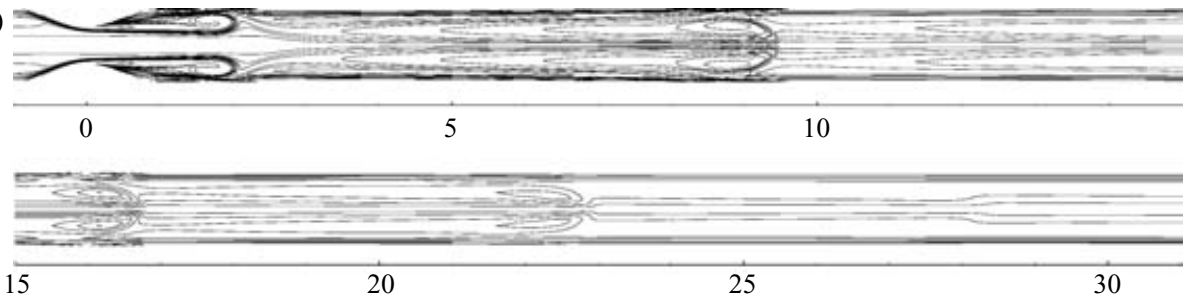

(b)
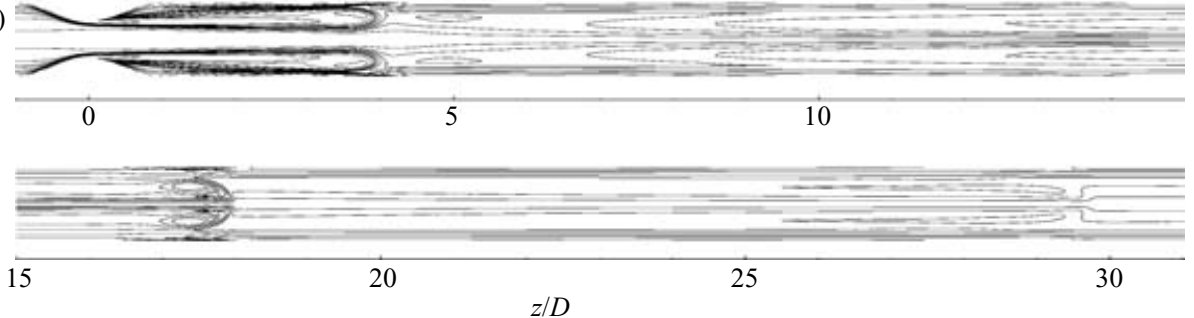

FIGURE 11. Azimuthal vorticity in the meridional plane of the axisymmetric base flow at the maximum point of the puslatile cycle for $(a) R e=400, U_{r e d}=2.5,(\alpha=15.9)$ and $(b) R e=415$, $U_{\text {red }}=5.0,(\alpha=11.4)$.

deceleration, minimum and mean acceleration instances of the sectionally averaged pulsatile velocity waveform.

The primary feature of the base flow is a plug-like jet which develops at the stenosis during the accelerating part of the cycle. At the front of this jet, a vorticity ring is generated that initially propagates down the pipe at a relatively constant speed despite the subsequent deceleration of the average flow.

In figure 10(a), we observe the early development of the plug-like jet, and the vorticity ring associated with the strong gradient in the axial flow at the front of the jet. At this point in the pulsatile cycle, the sectionally averaged velocity away from the stenosis is $\bar{u} / \bar{u}_{m}=1.75$. This can be compared with the flow immediately downstream of the emerging jet at $z / D=5$ in figure $10(a)$, where there is a centreline velocity of $u_{c} / \bar{u}_{m} \approx 3$, while in the core of the jet at $z / D=1.5$ the velocity is $u_{c} / \bar{u}_{m} \approx 7.8$. These values are consistent with the centreline velocities of the straight pipe solution which from equation (2.1) is $u_{c} / \bar{u}_{m}=2.8$ and the peak throat velocity which from mass conservation considerations is $4 \times 1.75 \bar{u}_{m}=7 \bar{u}_{m}$. From divergence considerations, we know that a high spatial gradient of the axial flow at the front of the jet is associated with a strong radial flow. The maximum magnitude of the radial flow is approximately twice $\bar{u}_{m}$ and occurs at $z / D \approx 5$.

From the vorticity plot of figure 10(a) we observe that the strong secondary flow due to the axial spatial gradient in velocity is associated with role up of the jet shear layer into a vortex ring. As the sectionally averaged flow decelerates and reaches its minimum (figures $10 c$ and $d$ ) the jet at the stenosis dies away, but the vortex ring continues to propagate down the pipe. An indication of the vortex ring strength can be implied by the centreline axial velocity at the location of the ring. The peak centreline axial velocity relative to the mean flow, $\bar{u}_{m}$, in the vicinity of the vortex ring is approximately $7.8,8.3,7.3$ and 7.4 in figures $10(a)$ to $10(d)$, respectively. Note that the mean sectional velocity ranges over $0.25 \leqslant \bar{u}_{m} \leqslant 1.75$.

The subsequent cycles generate similar vortex rings which continue to advect down the tube. Figure 11 shows the azimuthal vorticity at the maximum time instance 
(a)
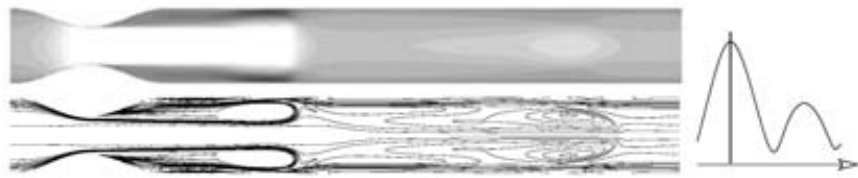

(b)
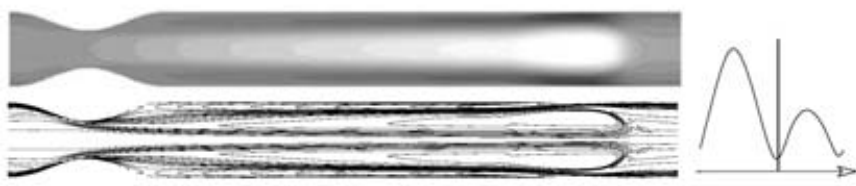

(c)
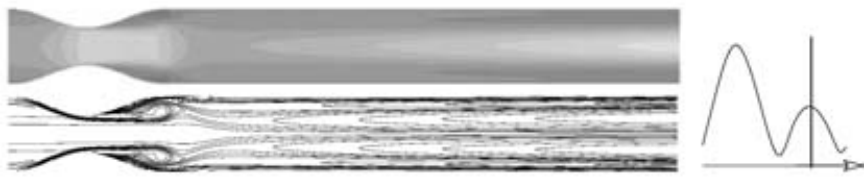

(d)
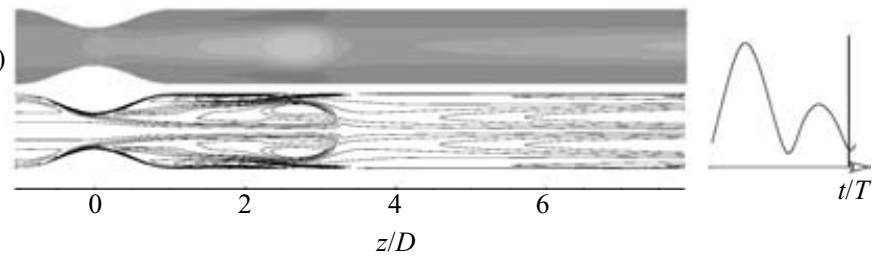

FIGURE 12. Streamwise velocity and azimuthal vorticity in the meridional plane of the axisymmetric base flow at four phases of the two-harmonic pulsatile cycle when $R e=425$, $U_{\text {red }}=5,(\alpha=11.5)$.

of the pulsatile cycle along a larger section of the pipe from $-1 \leqslant z / D \leqslant 31$ at $R e=400, U_{\text {red }}=2.5(\alpha=15.9)$. From this figure we observe that the intial separation between two sequential vortex rings is approximately $7 \mathrm{D}$. In figure $11(b)$, we show the azimuthal vorticity at $R e=415, U_{\text {red }}=5,(\alpha=11.4)$ using the same contour levels. For these parameters we observe that the separation between two sequential vortex rings has increased to approximately $14 \mathrm{D}$, which is consistent with the doubling of the period of the base flow cycle at this reduced velocity (all other parameters being held constant). Analogously, the separation at $R e=500, U_{\text {red }}=7.5(\alpha=10.2)$ was observed to be approximately $z / D=20$. Although the separation of the vortices seems to approximately scale with reduced velocity, the strength of the first vortex ring is notably higher when $U_{\text {red }}=2.5$. At $U_{\text {red }}=2.5$, the local peak vorticity in the vortex ring shown in figure $11(a)$ is approximately $60 \bar{u}_{m} / D$ as compared to approximately $45 \bar{u}_{m} / D$ when $U_{\text {red }}=7.5$ at the same point in the pulsatile cycle.

\subsubsection{Two-harmonic waveform}

For the two-harmonic waveform with $\alpha_{1}=0.75, \alpha_{2}=-0.75$ in equation (2.2) the base flow axial velocity and vorticity is shown in figure 12 at $R e=425, U_{\text {red }}=5$ $(\alpha=11.5)$. In this figure we have plotted the same time instances as a function of the time period previously considered in figure 10. As indicated on the right-hand side of this figure, the two-harmonic waveform could as an approximation be considered as two single-harmonic waveforms, the first having a peak magnitude of $\bar{u} / \bar{u}_{m}=2.5$ and the second having a peak of $\bar{u} / \bar{u}_{m}=1$.

The reduced velocity considered in figure 12 is double that of the case in figure 10 . All other constants being held fixed, a doubling of the reduced velocity can be 


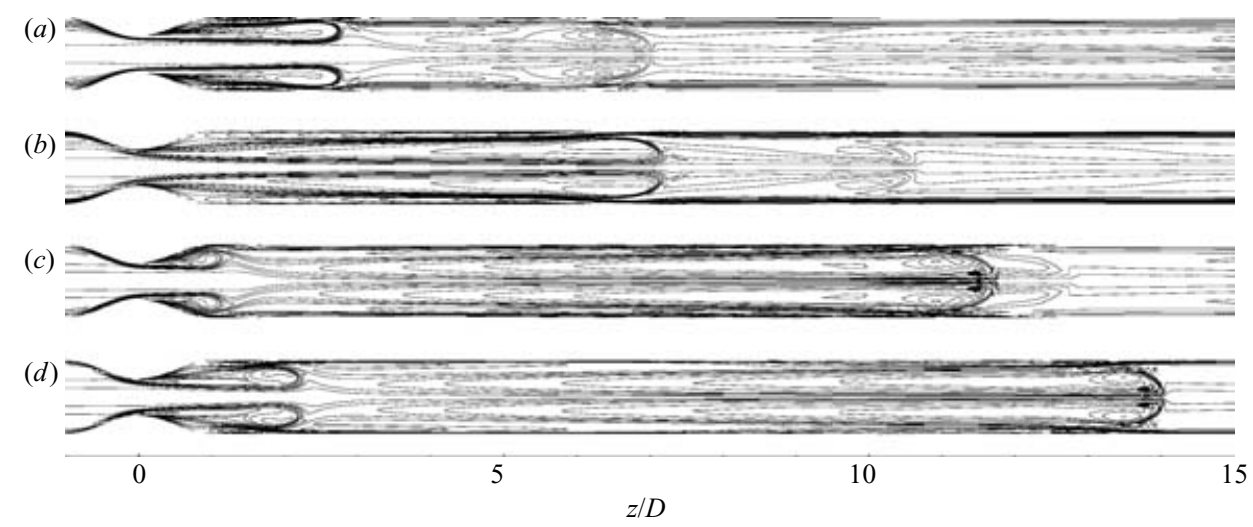

FIGURE 13. Contours of azimuthal vorticity in the meridional plane of the axisymmetric base flow at four phases of the puslatile cycle of the two-harmonic waveform with $R e=425, U_{\text {red }}=5$, $(\alpha=11.5) .(a-d)$ correspond to the instants shown in figure $12(a-d)$, respectively.

considered as a doubling of the time period $T$ between figure 10 and figure 12 . It is therefore possible to draw an analogy between each half-period of the waveform in figure 12 and the single harmonic waveform considered in figure 10. Under this analogy, it is appropriate to compare figure $12(a$ and $b)$ with figure $10(a$ and $c)$ since they represent the maximum and minimum time instances of the sectionally averaged velocity waveforms. However, in the two-harmonic waveform, the strength of the peak is now $\bar{u}_{\max }=2.5$ as opposed to 1.75 , and the peak centreline velocity at $z / D=2.5$ for the flow in figure $12(a)$ is $u_{c} / \bar{u}_{m}=10.6$. This increase in velocity naturally increases the strength of the vortex ring generated at the front of the jet. Further, this is consistent with the peak sectional velocity at the throat of the stenosis of $4 \times 2.5 \times \bar{u}_{m}=10 \bar{u}_{m}$. As shown in figure $12(c$ and $d)$ the smaller peak in the second half of the two-harmonic pulsatile cycle produces a second weaker vortex ring. The centreline velocity at $z / D=0.75$ at the instant of figure $12(c)$ is $4.5 \bar{u}_{m}$. Consistent with our previous comments, we observe that the reduced strength of the second vortex leads to a slower propagation down the pipe compared to the first vortex ring generated by the primary peak in the flow waveform.

Figure 13 shows the azimuthal vorticity in the meridional plane at the same phases as for figure 12 , but over the extended region $-1 \leqslant z / D \leqslant 15$. In figure $13(a)$ at $z / D=2$ and figure $13(b)$ at $z / D=6.5$ we observe the primary vortex ring corresponding to the dominant peak in the velocity waveform, propagating down the pipe. Also evident in these figures is the secondary vortex $(z / D=6.5$ in figure $13 a)$ generated by the second peak in the waveform from the previous cycle. In figure 13(c), we subsequently see the generation of a new secondary vortex ring at the stenosis throat associated with the second peak in the velocity waveform. However, at $z / D=11$, we also observe the amalgamation of the primary vortex ring from the current cycle with the secondary vortex ring from the previous cycle. The amalgamation leads to a weaker combined vortex ring in figure $13(d)$ where the maximum centreline velocity has decreased from 9.4 at $z / D=11$ in figure $13(c)$ to 7.7 at $z / D=13.5$ in figure $13(d)$.

\subsection{Floquet analysis}

In figure 14, we present summary results of Floquet stability analyses for a range of different pulsatile flows. This figure shows the largest Floquet multiplier $|\mu|_{\max }$ as a function of Reynolds number, Re, and lists the critical Reynolds number for each 


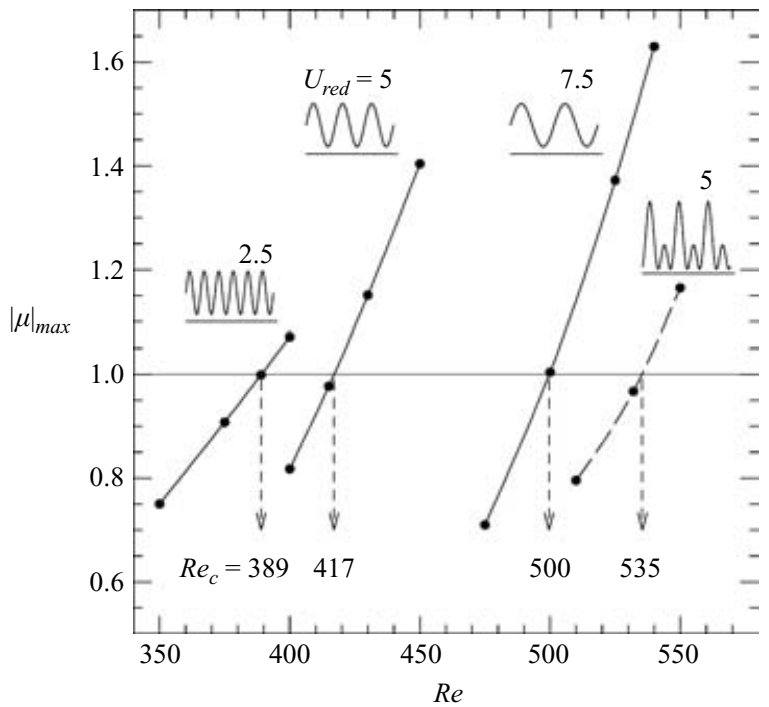

FIGURE 14. Plot of largest absolute Floquet multiplier, $|\mu|_{\max }$ as functions of Reynolds number for single-harmonic pulsatile flows with reduced velocities of $U_{\text {red }}=2.5,5$ and $7.5(\alpha=15.6$, 11.4 and 10.2, respectively) as well as for a two-harmonic waveform with $U_{\text {red }}=5$. In all cases, the leading Floquet multipliers are real and negative.

case. Also indicated by the inset plots within this figure are the corresponding pulsatile waveforms. It should be noted that for all the cases we have considered, the largest multipliers are real and negative: the leading modes all arise through period-doubling bifurcations with $\mu_{c}=-1$. Also, as was the case for the instability of the steady flow, the most unstable eigenmode always corresponds to the first azimuthal Fourier mode, $k=1$.

\subsection{Direct numerical simulation}

Following the Floquet analysis, we initiated DNS at $R e=400, U_{\text {red }}=2.5$ with a linear combination of the base flow and the most unstable eigenmode, projected to $N_{k}=16$ (32 planes of data in azimuth); figure 15 shows the evolution of kinetic energies in the azimuthal Fourier modes, analogous to figure 6 for the steady flow. Following an equilibration of energies at early times, there is an initial exponential growth phase (indicated by the dotted line based upon the Floquet analysis in the figure) that lasts until $t / T \approx 30$, after which the energy grows faster than exponentially until an initial nonlinear saturation takes place at $t / T \approx 40$. As was the case for the steady flow at $R e=750$, this faster-than-exponential growth indicates that the bifurcation is subcritical, although we have not followed this matter up in detail here. Succeeding this initial saturation, there follows a much longer transition to the asymptotic state at $t / T \sim 280$. In this state, the energies in the non-axisymmetric modes span approximately three orders of magnitude, as was the case for the steady flow DNS, but here the number of gridpoints is half, approximately 1.2 million.

\subsection{Discussion}

\subsubsection{Characteristics of linear instability modes}

In figure 16 we illustrate the characteristics of the base flow and leading eigenmode for the single-harmonic flow at $R e=400, U_{\text {red }}=2.5$, at a single phase $\left(t_{0}=0\right)$ in the pulse cycle. Figures $16(a)$ to $16(d)$ show contours of axial velocity component for the 


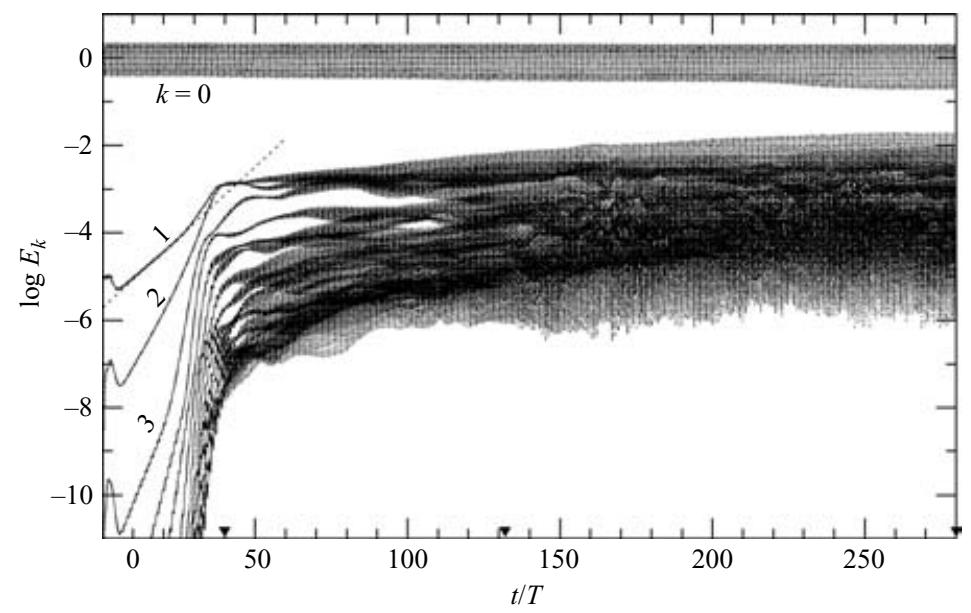

FiguRE 15. Growth to saturation of the pulsatile inlet flow solution at $U_{\text {red }}=2.5, R e=400$, approximately $3 \%$ above $R e_{c}$, represented by kinetic energies in azimuthal Fourier modes, with $N_{k}=16$. An initial exponential growth phase (indicated by the dotted line) is followed by faster than exponential growth near $t / T \sim 35$, an initial nonlinear saturation at $t / T \approx 40$, then a final slow growth to an aymptotic state, reached at $t / T \sim 250$.

(a)

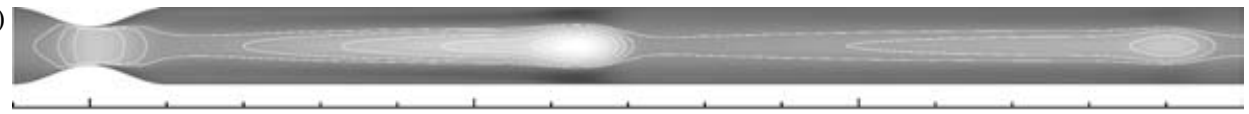

(b)

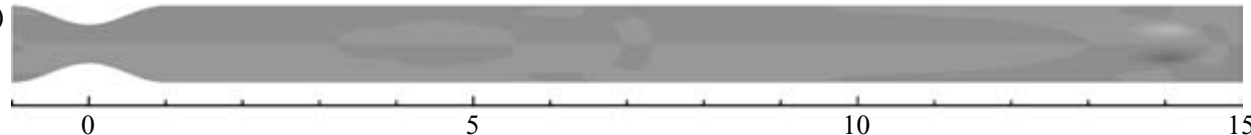

(c)

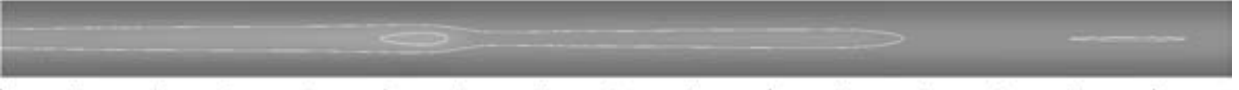

$(d)$

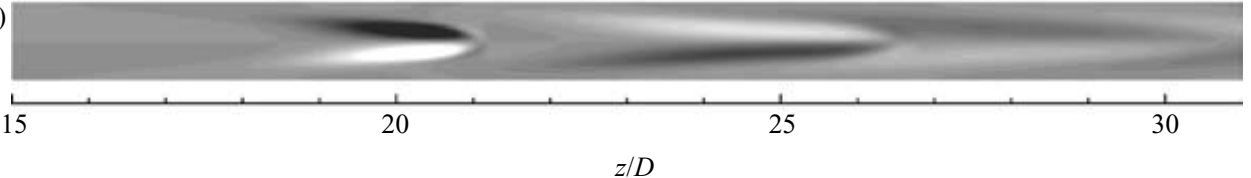

(e)

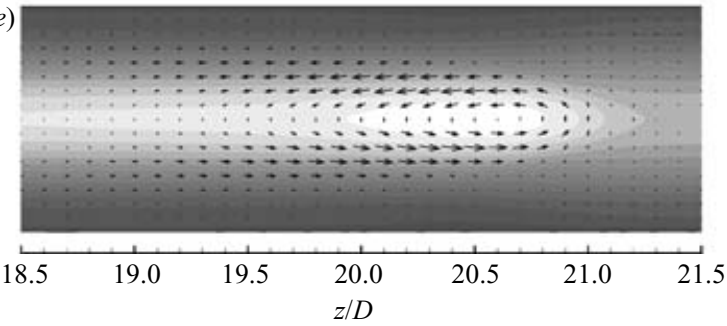

FIGURE 16. The base flow and the leading eigenmode for one phase in the flow cycle for the unstable pulsatile flow at $R e=400, U_{\text {red }}=2.5$, on a vertical centreplane. $(a, c)$ Contours of axial velocity of the base flow. $(b, d)$ Contours of axial velocity of the eigenmode. $(e)$ The velocity vectors of the eigenmode near its strongest streamwise location, superimposed on contours of axial velocity of the base flow. 
base flow, and the eigenmode, on a vertical plane bisecting the tube (i.e. the plane aligned with $\theta=0$ ). Within the length of tube represented, five base flow pulses can be discerned (the peak of the first pulse occurs at $z / D \approx 6.5$, while the last pulse is very weak).

As seen previously, the magnitude of the velocity pulse in the base flow falls with distance down the tube, as the flow re-adjusts to the Womersley flow conditions that would be found without the stenosis. For the first three base flow pulses, the separation remains relatively constant; however, after this point $(z / D \approx 20)$, the speed of propagation falls and the pulses become closer together. The strength of the Floquet mode initially increases with distance downstream, reaching a maximum at $z / D \approx 20$, before falling back as the strength of the pulses in the base flow decays. It can be seen that the sign of the perturbation velocity alternates on any axial traverse at a fixed radius: this is directly related to the period-doubling nature of the instability.

As highlighted in figure 16(e), the perturbation acts to provide an overturning moment on each velocity pulse of the base flow - and the sign of this moment alternates on successive pulses. The overturning flow can be seen in the perturbation velocity vectors. In terms of vorticity, the perturbation tends to alternately tilt the vortex rings that are ejected from the stenosis during each successive pulse: the downwash from the tail of the instability that grows on any one ring induces the opposite tilt in its successor. The influence of this alternating tilting will also be seen in the DNS results to be presented in figure 17, as will be discussed in $§ 6.4 .2$. We note that this type of instability is distinct from those relevant to flows in constricted two-dimensional channels, where 'vorticity waves' and shear-layer instabilities are observed (Sobey 1985; Pedley 2000). However, the nature of the instability does bear some similarity to the bifurcation of free jets observed by Glezer (see Saffman 1981) and Reynolds et al. (2003).

As the reduced velocity for the single-harmonic waveform is increased from $U_{\text {red }}=2.5$ to 5 and then 7.5 , we recall that the pulse separations in the base flows also increase. However, in the latter two cases the peak eigenmode magnitude is associated with the second rather than the third pulse, as occurs when $U_{\text {red }}=2.5$. We recall that the initial strength of the first vortex ring grows weaker as $U_{\text {red }}$ is increased. In contrast, for the two-harmonic waveform where $U_{\text {red }}=5$ the peak eigenmode levels are associated with the third pulse from the stenosis. Although the pulse separations for the single- and double-harmonic waveforms are similar when $U_{\text {red }}=5$ (see figures 11 and 13), the initial vortex is much stronger, having approximately double the peak vorticity in the two-harmonic waveform case. We therefore observe that the initial strength of the vortex ring may dictate how far the vortex ring must propagate while decaying in strength before the period doubling instability dominates. The alignment of the peak eigenmode energy with the third rather than the second pulse-front in the case of the two-harmonic inflow waveform also explains the need for the longer-outflow domain (figure $3 c$ ) for this investigation.

Finally we note that, with all other parameters fixed, the shorter the time period of the oscillation, the smaller the reduced velocity. Equivalently, the higher the Womersley number, then the lower will be the critical Reynolds number, as is indicated by the values presented in figure 14. If the instability is promoted by an interaction between subsequent pulses, then the increased separation at higher $U_{\text {red }}$ may require higher Reynolds numbers in order to overcome viscous damping on the velocity induced by one vortex ring at the location of another, and allow unstable growth to occur. The trend of higher reduced velocity leading to a lower critical 
(a)

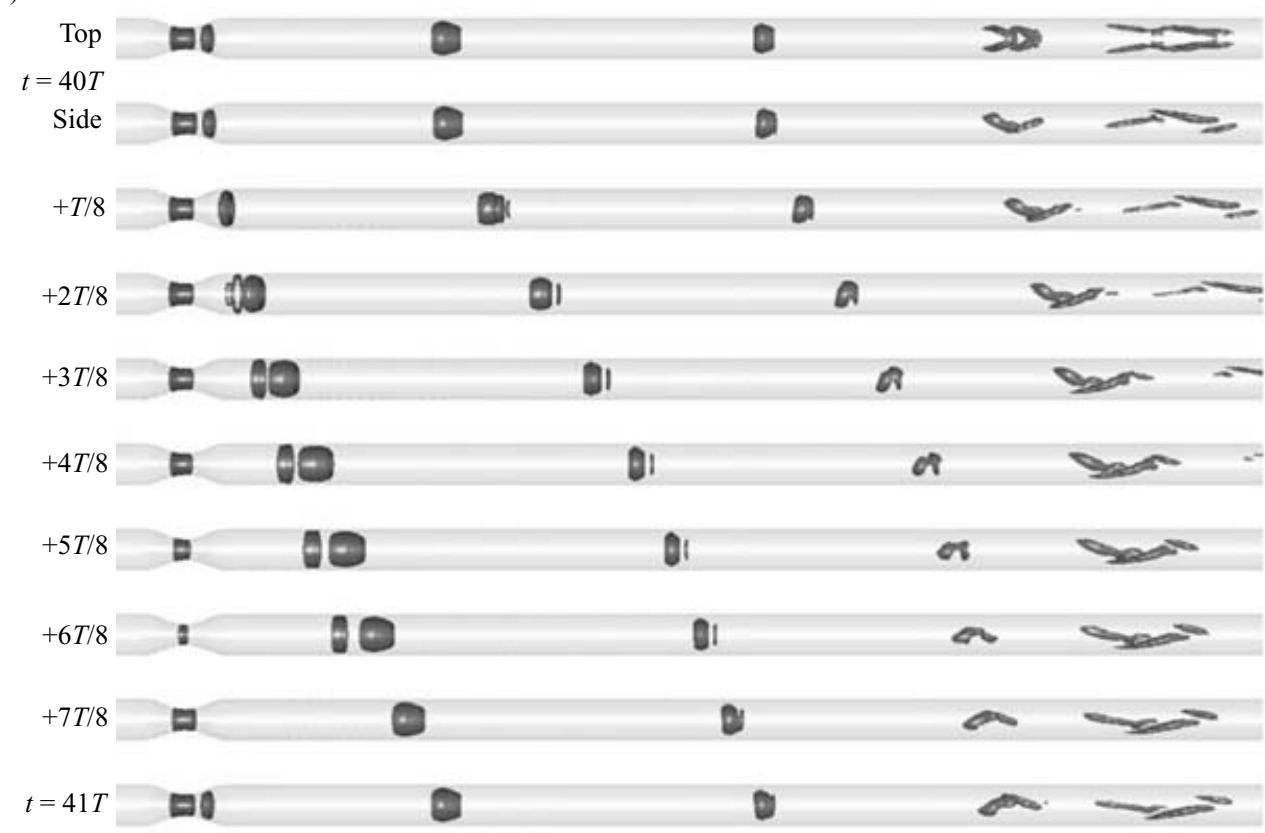

(b)

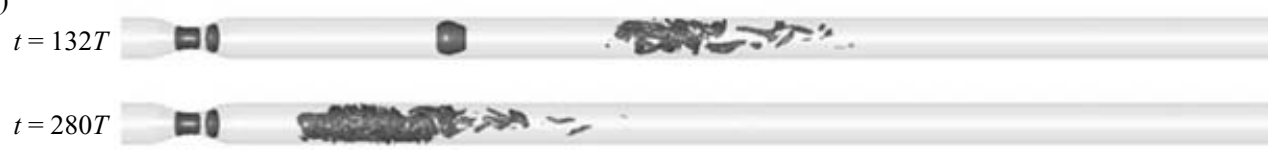

(c)

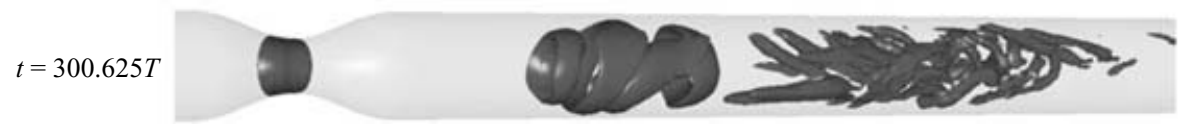

FIGURE 17. Visualizations for the DNS of pulsatile flow at $U_{\text {red }}=2.5, R e=400$. Isosurfaces are extracted using the $\lambda_{2}$ criterion of Jeong \& Hussain (1995). (a) A sequence of visualizations over one pulse cycle, commencing just after the initial saturation seen at $t / T=40$ in figure 15 . Note that the images at $t=40 T$ and $t=41 T$ have almost exact reflection symmetry about the tube centre-plane, consequent on the period-doubling bifurcation of the instability mode. (b) Two visualizations at later times in the progression to the asypmtotic state. Note the upstream movement of breakdown. (c) A detailed perspective view of the breakdown of vortex rings in the cycle following $t=300 T$. Downstream of the stenosis, the first group of structures shows a ring deforming during the final stages of the tilting process, while the second group shows the decaying breakdown of the previous ring.

Reynolds number is contrary to the experimental observation of Nerem, Seed \& Wood (1972) who studied the transition to turbulence in the aorta of a dog, in the absence of a stenosis. Significant differences between the two studies occur in the geometric and inflow conditions which could reasonably lead to the alternative trends in critical Reynolds number with reduced velocity.

\subsubsection{Nonlinear saturation and transition}

Figure 17 illustrates the flow at various stages of the growth indicated in figure 15. The instantaneous isosurfaces show a negative contour of $\lambda_{2}$, the intermediate eigenvalue of the symmetric tensor $\boldsymbol{S}^{2}+\boldsymbol{\Omega}^{2}$, with $\boldsymbol{S}$ and $\boldsymbol{\Omega}$ being, respectively, the 
symmetric and anti-symmetric parts of the velocity gradient tensor $\nabla \boldsymbol{u}$ - this measure was introduced by Jeong \& Hussain (1995) for identification of vortical structures in three-dimensional flows.

The two initial panels of figure 17(a) show top and side views of the isosurface at time instant $t=40 T$ (see figure 15 for the time base). We find a number of groups of structures (each of which, near to the stenosis, can be thought of as being associated with a vortex ring) that correspond to the evolution of individual velocity pulses. The following eight panels show snap-shots at $T / 8$ phases up to $t=41 T$ : taken together, these panels represent the evolution over time interval $T$ of the flow following the initial saturation in figure 15. The relative coherence of the structures shows that at this time, the flow is still quite well-organized, and (as evidenced by comparison of the top and side views) retains the symmetry of the initial condition. Looking at the side views in this sequence, we can observe how, travelling downstream, a vortex-ring structure first acquires a small tilt (corresponding to perturbation by the Floquet eigenmode), then, presumably through a process of self-induction and wall interaction, is rapidly torn apart until it appears as a packet of lambda-vortices. Note that at $t=41 T$, we see almost exactly a vertical reflection of the structures seen in side view at $t=40 T$; this is a consequence of the fact that the instability arises through a period-doubling bifurcation.

The two panels of figure $17(b)$ show the flow at $t=132 T$ and $t=280 T$, respectively, in the midst of and at the end of the slow final growth in energies of the nonaxisymmetric modes observed in figure 15 . We find that this slow transition has come about as the vortex-ring breakdown, seen near the end of the field of view in the initial panels, moves progressively further upstream. In the asymptotic state, this breakdown takes place almost immediately after ring formation, and is highly turbulent.

At $t=280 T$, the flow still has the symmetry of the initial conditions, as expressed in (3.9). Before proceeding with further evolution, the real and imaginary parts of the first azimuthal mode were perturbed with Gaussian-distributed noise, at $O\left(10^{-4}\right)$, which, during further evolution to $t=300 T$, led to the redistribution of energy between the real and imaginary components of the modes, and destruction of symmetry. However, this redistribution scarcely affected the asymptotic distribution of energies between the modes observed near $t=280 T$ in figure 15 . Also, when the nature of the flow is considered at the largest length scales and over a moderate number of cycles, it does not substantially perturb the period-doubling mechanism, indicating that it is comparatively robust.

Figure $17(c)$ shows a perspective view of structures near the stenosis, part-way through the cycle following $t=300 T$, again visualized using an isosurface of $\lambda_{2}$. In the parallel section of the tube downstream of the stenosis, there are two groups of structures. The upstream group shows a single vortex ring in the late stages of its tilting deformation; it is evident that this is a complex three-dimensional event. The second group of structures represents the decaying breakdown of the previous vortex ring. Note that the streamwise spacing of successive breakdowns is much reduced compared to those seen at earlier times $t / T=41,132$, in figure 17 .

The process of ring breakdown is highly dissipative, and will lead to high levels of wall shear stress, as well as of its temporal and spatial gradients, all of which are now thought to be relevant to development of atheroma and thrombi. It is interesting that, even though the linear stability analysis and the initial nonlinear saturation condition $(t \approx 40 T)$ shows the breakdown to occur far downstream of the stenosis in our idealized geometry, it subsequently progresses upstream to occur only a few diameters downstream of the stenosis. It should also be pointed out, however, that the 
highest wall shear stresses (while perhaps not their highest spatio-temporal gradients) will almost certainly occur in the throat of the stenosis itself.

\subsubsection{Shear-layer instability}

In experimental investigations of stenotic flow with geometries similar to those considered in this paper (Cassanova \& Giddens 1978; Ahmed \& Giddens 1983), the breakdown in the post-stenotic region has been accompanied by finer-scale shearlayer-type instabilities, as was discussed in relation to steady inlet flows in $\$ 5.4 .4$. In the saturated nonlinear state with a steady inflow, we have observed shear-layer instabilities as shown in figure 9. During our DNS study of the single-harmonic pulsatile flow, on the other hand, we did not observe signs of shear-layer instabilities.

Nevertheless, during our resolution studies for computing pulsatile base flows to be used in Floquet analysis, shear-layer oscillations were often noted when the flows were under-resolved. Localized oscillations produced high-frequency signals, readily observed in pressure time series at sampling points remote from the instability site. These instabilities were usually found to be triggered at times when a pulsatile velocity front propagated through a region of low resolution. The oscillations always disappeared when mesh resolution was sufficient - in fact, oscillations became one of our key diagnostics, indicative of poor mesh design or under-resolution.

The physical experiments, our experience in computing base flows, and some of the DNS results described here all suggest that in this problem, jet shear layers may be subject to convective instability (Huerre \& Monkewitz 1985, 1990). While this is not our main theme, and difficulties of analysis (particularly considering that we are confronted with pulsatile flows) preclude a rigorous examination, we describe in this section a brief heuristic study of shear-layer oscillations for the axisymmetric two-harmonic base flow at $U_{\text {red }}=5, R e=550$. Of all the cases dealt with, this was the most susceptible to oscillation, probably because of its elevated Reynolds number and peak-to-mean ratio, the combination of which results in thin shear layers.

The investigation was computed at $N_{p}=9$ on the mesh shown in figure $3(c)$; at this resolution, and with inlet flows of the form (2.2), oscillations in the flow are not observed. We then added a small high-frequency component to the inlet flow, now described by

$$
\bar{u}(t)=1+0.75 \sin (2 \pi t / T)-0.75 \cos (4 \pi t / T)+A_{m p} \sin \left(n_{f} 2 \pi t / T\right),
$$

and where we have taken $A_{m p}=0.001$, i.e. a maximum perturbation amplitude which is $1 / 1000$ of the mean base flow velocity, and $n_{f}$ is an adjustable integer harmonic.

Figure 18( $a$ ) illustrates the periodic time series of perturbation axial velocity, $\Delta u$, downstream of the stenosis $(z / D=1, r / D=0.25)$ for different values of the harmonic $n_{f}$. The perturbation velocity, $\Delta u$, was evaluated by subtracting the time history of the unforced inflow solution $\left(A_{m p}=0\right)$ from the forced inflow $\left(A_{m p}=0.001\right)$. Figure $18(b)$ shows the amplification of inflow forcing, evaluated by the maximum perturbation velocity, $|\Delta u|_{\max }$ at a fixed point defined as

$$
|\Delta u|_{\max }(z, r)=\max _{0 \leqslant t<T}|\Delta u(z, r, t)|,
$$

normalized by the forcing amplitude $A_{m p}$. Here we observe that the maximum amplification of the inflow forcing occurs at a frequency corresponding to $n_{f}=65$, where we have an amplification of $|\Delta u|_{\max }=272 A_{m p}$. In figure 18(c), we show contours of vorticity in the base flow at $t / T \approx 43 / 128$, which corresponds to the phase at which the perturbation velocities are greatest. Figure $18(d)$ shows vorticity at the same phase in the perturbed case for $n_{f}=65$. The shear layers immediately trailing the primary 

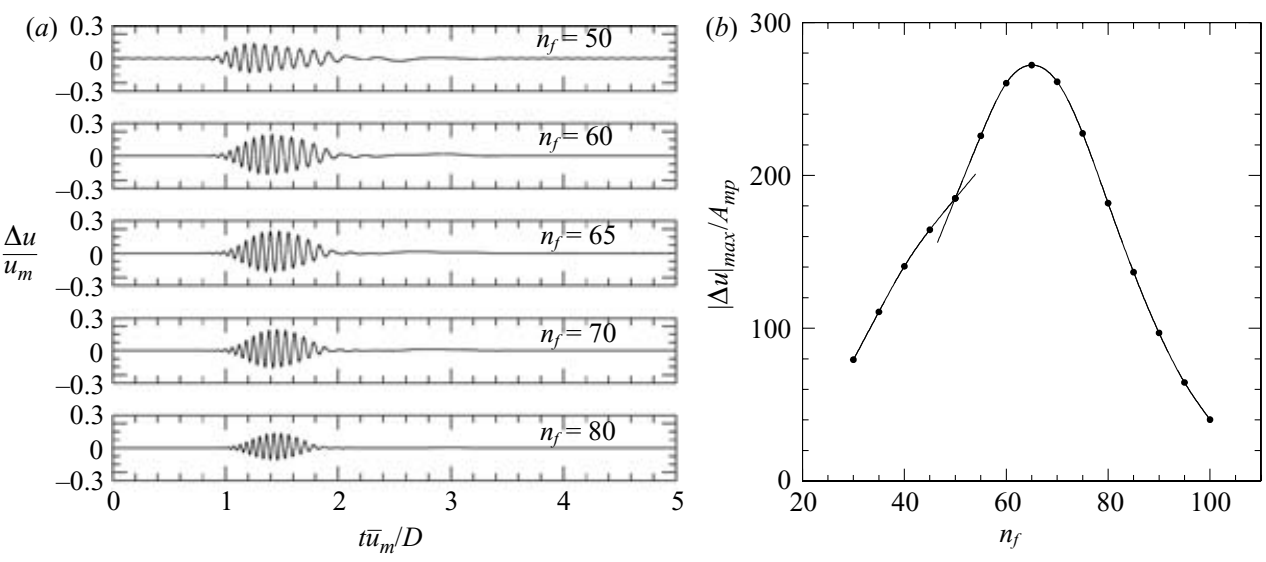

(c)
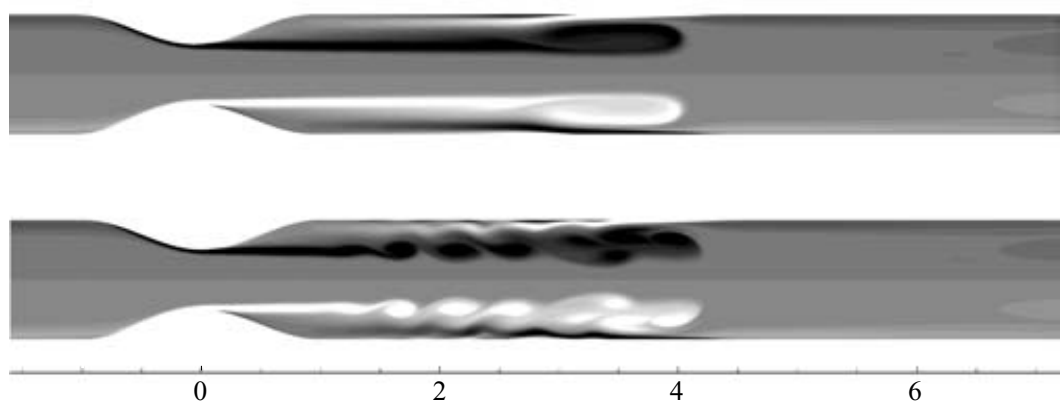

$(d)$

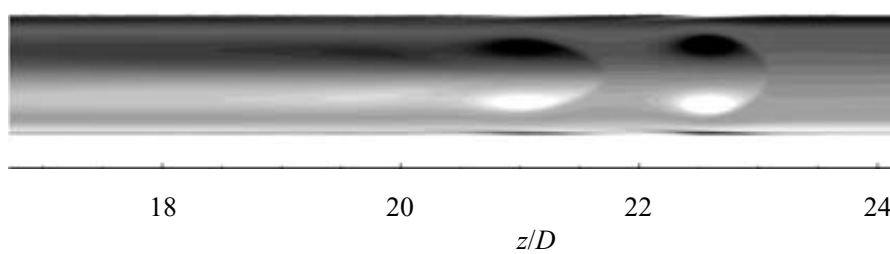

FIGURE 18. Magnitude of shear-layer oscillations in the base flow for the two-harmonic case at $R e=550$, with an additional high-frequency perturbation, $A_{m p}=0.001$, imposed at the domain inlet. The data are for the axial component of perturbation velocity at a single sampling point, location $(z / D=1, r / D=0.25)$. The dependence on the perturbation frequency $n_{f}$ of the perturbation time-histories and the maximum normalized perturbations are shown in $(a)$ and (b), respectively. $(c, d)$ Vorticity at $t / T \approx 43 / 128$ for the unforced base flow and the case when $n_{f}=65$. (The last panel uses a different isocontour scaling to the previous two.)

vortex ring clearly display a Kelvin-Helmholtz-type instability which also, at this level of $A_{m p}$, appears to disrupt the primary vortex. However, further downstream at $z / D=$ 20, where the Floquet mode is at its most energetic, we observe that the shear-layer oscillations have decayed, leaving a vorticity pattern similar to the unforced case.

The oscillations bear the classic hallmarks of convective instability: they are highly receptive in a well-defined frequency band, and do not develop in the absence of forcing. In animations, they are clearly seen to grow as they propagate downstream. On the other hand, here at least, the instability exists only over part of the base flow cycle, and does not survive into the region where the three-dimensional instabilities develop. The question of how much influence convective instability might ultimately have on large-scale flow structures produced by the primary absolute instability in 
these flows remains an open one. This point also depends on the size and nature of any perturbation. However, we have calculated the Floquet multiplier at a Reynolds number of $R e=550$ and a polynomial order of $N_{p}=9$ for a base flow with inflow forcing at a frequency of $n_{f}=65$ and amplitude $A_{m p}=0.001$ (as for the base flow illustrated in figure 18). For this case, we again obtained period doubling, with a multiplier of $\mu=-1.180$ as opposed to $\mu=-1.174$ for the case with no highfrequency forcing.

\subsubsection{Comparison to previous studies}

The most relevant works for comparison purposes are the flow visualization and laser Doppler measurements of Ahmed \& Giddens $(1983,1984)$ and Ahmed (1998), and the flow visualization results of Ojha et al. (1989). Ahmed \& Giddens have $R e=600, U_{p m} \approx 1.7$ and $\alpha=7.5$, and the parameters of Ojha et al. are similar, with $R e=575, U_{p m} \approx 1.6$ and $\alpha=7.5$. Both sets of experiments used an approximation to a single-harmonic pulsatile waveform. Both works employ (at least in part) a convergent/divergent axisymmetric stenosis with $75 \%$ occlusion, but that of Ojha et al. is sharp-edged while Ahmed \& Giddens's is smooth. For $75 \%$ stenosis, highly unsteady but transient fluctuations were observed downstream of the tube constrictions in both experiments.

The lowest Womersley number investigated for the onset of linear instability in the present work is $\alpha=10.2\left(U_{\text {red }}=7.5\right)$ where $R e_{c}=500$, and we would expect that the lower value of $\alpha=7.5$ used by the other workers would result in a higher critical Reynolds number, probably of order 600 or above. On the other hand, it appears quite possible (on the basis of the DNS investigations for the steady flow and for the $U_{\text {red }}=2.5$ pulsatile flow) that the bifurcation would be subcritical in all cases, so we might also expect that three-dimensional unsteadiness could be sustained down to the Reynolds numbers found in the above experiments.

In Ahmed \& Giddens (1984) it is stated that 'turbulence was found only for the $75 \%$ stenosis and was created only during part of the cycle', whereas in Ahmed (1998) these fluctuations, which are strongest for $2.5<z / D \leqslant 6$, are characterized as non-turbulent owing to the presence in the conditional velocity spectra of a band of dominant frequencies associated with 'vortex shedding and a turbulent front'. These findings are in quite good agreement with the dye-front flow visualization and interpretation provided by Ojha et al. (1989). For the $75 \%$ stenosis, they found four post-stenotic zones: Zone I, reaching to $z / D=3$, is called the 'stable jet region', although some indication of (apparently axisymmetric) wavy structure can be observed on the jet front in this region; Zone II, $3<z / D \leqslant 4.5$ is called the 'transition region', where the waves become larger; in Zone III, the 'turbulent region', $4.5<z / D \leqslant 7.5$, the front rapidly distorts; Zone IV, $z / D>7.5$ is labelled 'relaminarization'.

These experimental results are thus in reasonable agreement with the kind of asymptotic behaviour we have observed in DNS, for $R e=400$ and $\alpha=15.9$, as can be seen in figure $17(b, c)$ : a rapid distortion of a vortex ring becoming evident a few diameters downstream of the stenosis, leading to a highly unsteady/transitional breakdown at $z / D \sim 6$, following which, relaminarization takes place further downstream.

\section{Conclusions}

We have studied steady and pulsatile flow in a smooth axisymmetric $75 \%$ stenotic tube, using a combination of three-dimensional stability analysis and direct numerical 
simulation. Our objective here has been to establish stability characteristics and overall flow behaviour, rather than to provide detailed statistics.

For steady inflow, we have observed a linear instability at $R e_{c}=722$, where the Reynolds number is based upon the sectionally and time-averaged flow, $\bar{u}_{m}$ and the full pipe diameter, $D$. The instability arises in the $k=1$ azimuthal Fourier mode and has the character of a Coanda-type wall attachment, where the axisymmetric shear layer of the base flow generated in the post-stenotic region is perturbed towards the pipe wall. The nature of the initial nonlinear saturation observed in DNS $R e=750$ indicates that the instability is subcritical and therefore hysteretic in nature. On further evolution, the flow becomes turbulent downstream of the stenosis. Taking this state as an initial condition and reducing the Reynolds number, we have observed turbulent flow down to $R e=688$, confirming the presence of hysteresis. The asymptotic state of the flow at $R e=750$ contains long-period fluctuations on a time scale of $35 \mathrm{D} / \bar{u}_{m}$. These fluctuations are associated with a slow flapping of the jet, and coupled axial movement of a region of localized turbulent breakdown, the spatial onset of which oscillates between approximately $4 D$ and $10 D$ from the throat of the stenosis. The critical Reynolds numbers observed through this numerical analysis are consistent with the experimental work of Cassanova \& Giddens (1978) and Ahmed \& Giddens (1983).

For pulsatile inflows, we have considered two types of non-reversing pulsatile waveforms which satisfy the Womersley solution for straight tubes. The first waveform contained a mean and single harmonic with a peak to mean ratio of $U_{p m}=1.75$ and where we have used three different pulse periods $T$, as expressed by reduced velocities of $U_{\text {red }}=\bar{u}_{m} T / D=2.5,5$ and 7.5. A further waveform of a mean and two harmonics was also considered, with a peak-to-mean ratio of $U_{p m}=2.5$ and at a reduced velocity of $U_{\text {red }}=5$. Floquet stability analysis was undertaken for these four inlet-flow waveforms. For the single-harmonic inflows with $U_{\text {red }}=2.5,5$ and $7.5, R e_{c}=389,417$ and 500 (giving critical Womersley numbers of 15.6, 11.4 and 10.2), respectively. The critical Reynolds number for the two-harmonic waveform at $U_{\text {red }}=5$ was found to be $R e_{c}=535$. Waveforms with a shorter time period (and reduced velocity) are more unstable. Somewhat unexpectedly, the two-harmonic waveform, which had a higher peak-to-mean ratio, was found to be more stable than the single-harmonic waveform at the same reduced velocity. An interesting feature of the two-harmonic flow is amalgamation of the two vortex rings of different initial strength that are generated in one pulse cycle. For all the pulsatile cases considered, instability occurred through a period-doubling bifurcation, and the nature of the instability was identified as alternating tilting of vortex rings generated during sequential pulsatile cyclic ejections from the stenotic constriction. This behaviour appears to bear some similarity to the bifurcations of free jets observed by Glezer (see Saffman 1981) and Reynolds et al. (2003), although here constrained by the presence of tube walls.

Direct numerical simulation was also performed for the single harmonic waveform at $U_{\text {red }}=2.5$ and $R e=400$. This simulation demonstrated that the instability was again subcritical and that after a linear instability phase which tilts the vortex ring ejected from the stenosis, it rapidly breaks down into finer-scale components. After a relatively long period $(O(200 T))$ the nonlinear saturated state develops to produce a localized turbulent breakdown which occurs at approximately $4 D$ downstream of the stenosis. Although this characteristic is similar to the final state of the steady flow, we note that the instability and transition mechanisms are very different and occur at a lower average Reynolds number in the pulsatile flows.

While computing axisymmetric base flow for the two-harmonic waveform, shearlayer instabilities were observed when the simulations were under-resolved. Motivated 
by this observation and evidence of shear-layer oscillations in some of the available experiments, we have also performed a non-exhaustive investigation of shear-layer instability by perturbing the fully resolved axisymmetric base flow on the inflow boundary with high-frequency components of amplitude $1 / 1000$ that of the mean flow speed. A maximum amplification of approximately 275 was observed at a fixed point in the shear layer just downstream of the stenosis when the forcing frequency was on the 65th harmonic of the base flow fundamental frequency. However, at this level of perturbation the shear-layer oscillations, while of moderate magnitude, remain spatially localized enough to not greatly influence Floquet instability.

The motivation for this study derives from interest in the flows of the human arterial system. For this idealized geometry, the steady and puslatile flow critical Reynolds numbers predicted here are relatively high for physiological conditions in the systemic or coronary arteries, although the reduced velocities of the pulsatile flows are reasonable. However, in an axisymmetric stenosis, the sectional Reynolds number increases as the inverse of the local diameter. We might therefore expect that higher degrees of stenosis (i.e. greater reduction of diameter) would lead to a reduction of the critical Reynolds number. Further, the subcritical nature of the instabilities implies that they are hysteretic and so turbulent flow can persist at lower Reynolds numbers than the critical value as demonstrated by the direct numerical simulations. The final saturated states of the nonlinear breakdown of both the steady and pulsatile flows studies also indicate regions of strong localized turbulence which are associated with strong temporal and spatial shear stress gradients that have been implicated in the onset of arterial disease such as atherosclerosis.

This work was supported through the Merit Allocation Scheme of the Australian Partnership for Advanced Computing. S. J.S. would like to acknowledge financial support from the Royal Academy of Engineering in the form of a Global Research Award. H. M. B. wishes to acknowledge EPSRC grant GR/T02553/01 in financial support of a Visiting Fellowship.

\section{REFERENCES}

AHMED, S. A. 1998 An experimental investigation of pulsatile flow through a smooth constriction. Exptl Thermal Fluid Sci. 17, 309-318.

Ahmed, S. A. \& Giddens, D. P. 1983 Velocity measurements in steady flow through axisymmetric stenoses at moderate Reynolds numbers. J. Biomech. 16, 505-516.

Ahmed, S. A. \& Giddens, D. P. 1984 Pulsatile poststenotic flow studies with laser Doppler anemometry. J. Biomech. 17, 695-705.

Akhavan, R., Kamm, R. D. \& Shapiro, A. H. 1991 An investigation of transition to turbulence in bounded oscillatory Stokes flows. Part 1. Experiments. J. Fluid Mech. 225, 395-422.

BarkLey, D. \& Henderson, R. D. 1996 Three-dimensional Floquet stability analysis of the wake of a circular cylinder. J. Fluid Mech. 322, 215-241.

Berger, S. A. \& Jou, L.-D. 2000 Flows in stenotic vessels. Annu. Rev. Fluid Mech. 32, 347-384.

BlackBURN, H. M. 2002 Three-dimensional instability and state selection in an oscillatory axisymmetric swirling flow. Phys. Fluids 14, 3983-3996.

BlackBURN, H. M. \& Lopez, J. M. 2003a On three-dimensional quasi-periodic Floquet instabilities of two-dimensional bluff body wakes. Phys. Fluids 15, L57-60.

BLACKBURN, H. M. \& LOPEZ, J. M. $2003 b$ The onset of three-dimensional standing and modulated travelling waves in a periodically driven cavity flow. J. Fluid Mech. 497, 289-317.

BlackbuRn, H. M. \& Sherwin, S. J. 2004 Formulation of a Galerkin spectral element-Fourier method for three-dimensional incompressible flows in cylindrical geometries. J. Comput. Phys. 197, 759-778. 
Caro, C. G., Fitz-Gerald, J. M. \& Schroter, R. C. 1971 Atheroma and arterial wall shear: observation, correlation and proposal of a shear dependent mass transfer mechanism for atherogenesis. Proc. R. Soc. Lond. B 177, 109-159.

Cassanova, R. A. \& Giddens, D. P. 1978 Disorder distal to modified stenoses in steady and pulsatile flow. J. Biomech. 11, 441-453.

Huerre, P. \& Monkewitz, P. A. 1985 Absolute and convective instabilities in shear layers. J. Fluid Mech. 159, 151-168.

Huerre, P. \& Monkewitz, P. A. 1990 Local and global instabilities in spatially developing flows. Annu. Rev. Fluid Mech. 22, 473-537.

JeOng, J. \& Hussain, F. 1995 On the identification of a vortex. J. Fluid Mech. 285, 69-94.

Khalifa, A. M. A. \& Giddens, D. P. 1981 Characterization and evolution of poststenotic disturbances. J. Biomech. 14, 279-296.

Ku, D. N. 1997 Blood flow in arteries. Annu. Rev. Fluid Mech. 29, 399-434.

Long, Q., Xu, X. Y., Ramnarine, K. V. \& Hoskins, P. 2001 Numerical investigation of physiologically realistic pulsatile flow through arterial stenosis. J. Biomech. 34, 1229-1242.

McDonald, D. A. 1974 Blood Flow in Arteries, 2nd edn. Edward Arnold.

Mallinger, F. \& DrikaKis, D. 2002 Instability in three-dimensional, unsteady, stenotic flows. Intl J. Heat Fluid Flow 23, 657-663.

Mills, C. J., Gabe, I. T., Gault, J. H., Mason, D. T., Ross JR, J., Braunwald, E. \& Shillingford, J. P. 1970 Pressure-flow relationships and vascular impedance in man. Cardiovasc. Res. 4, 405-417.

Nerem, R. M., Seed, W. A. \& Wood, N. B. 1972 An experimental study of the velocity distribution and transition to turbulence in the aorta. J. Fluid Mech. 52, 137-160.

Ojha, M., Cobbold, R. S. C., Johnston, K. W. \& Hummel, R. L. 1989 Pulsatile flow through constricted tubes: an experimental investigation using photochromic tracer methods. J. Fluid Mech. 203, 173-197.

Pedley, T. J. 2000 Blood flow in arteries and veins. In Perspectives in Fluid Dynamics: A Collective Introduction to Current Research (ed. G. K. Batchelor, H. K. Moffatt \& M. G. Worster), chap. 3, pp. 105-158. Cambridge University Press.

Reynolds, W. C., Parekh, D. E., Juvet, P. J. D. \& Lee, M. J. D. 2003 Bifurcating and blooming jets. Annu. Rev. Fluid Mech. 35, 295-315.

SAAD, Y. 1992 Numerical Methods for Large Eigenvalue Problems. Wiley.

Saffman, P. G. 1981 Dynamics of vorticity. J. Fluid Mech. 106, 49-58.

SobeY, I. J. 1985 Observation of waves during oscillatory channel flow. J. Fluid Mech. 151, 395-426.

Sobey, I. J. \& Drazin, P. 1986 Bifurcations of two-dimensional channel flows. J. Fluid Mech. 171, $263-287$.

Stroud, J. S., Berger, S. A. \& Saloner, D. 2002 Numerical analysis of flow through a severely stenotic carotid artery bifurcation. Trans. ASME K: J. Biomech. Engng 124, 9-20.

TAYLOR, C. A. \& Draney, M. L. 2004 Experimental and computational methods in cardiovascular fluid mechanics. Annu. Rev. Fluid Mech. 36, 197-231.

Tuckerman, L. S. \& BARKLEY, D. 2000 Bifurcation analysis for timesteppers. In Numerical Methods for Bifurcation Problems and Large-Scale Dynamical Systems (ed. E. Doedel \& L. S. Tuckerman), pp. 453-566. Springer.

Wilhelm, D. \& KLEISER, L. 2001 Stability analysis for different formulations of the nonlinear term in $P_{N}-P_{N-2}$ spectral element discretizations of the Navier-Stokes equations. J. Comput. Phys. 174, 306-326.

WomersLey, J. R. 1955 Method for the calculation of velocity, rate of flow and viscous drag in arteries when the pressure gradient is known. J. Physiol. 127, 553-563.

Wootton, D. M. \& Ku, D. N. 1999 Fluid mechanics of vascular systems, diseases, and thrombosis. Annu. Rev. Biomed. Engng 1, 299-329.

YANG, W. H. \& Yıн, C.-S. 1977 Stability of time-periodic flows in a circular pipe. J. Fluid Mech. 82, 497-505.

ZANG, T. A. 1991 Numerical simulation of the dynamics of turbulent boundary layers: perspectives of a transition simulator. Phil. Trans. R. Soc. Lond. A 336, 95-102. 